TRANSACTIONS OF THE

AMERICAN MATHEMATICAL SOCIETY

Volume 359, Number 3, March 2007, Pages 1129-1160

S 0002-9947(06)04039-6

Article electronically published on September 11, 2006

\title{
RELATIVE WEAK COMPACTNESS \\ OF ORBITS IN BANACH SPACES ASSOCIATED WITH LOCALLY COMPACT GROUPS
}

\author{
COLIN C. GRAHAM AND ANTHONY T. M. LAU
}

\begin{abstract}
We study analogues of weak almost periodicity in Banach spaces on locally compact groups.

i) If $\mu$ is a continous measure on the locally compact abelian group $G$ and $f \in L^{\infty}(\mu)$, then $\{\gamma f: \gamma \in \widehat{G}\}$ is not relatively weakly compact.

ii) If $G$ is a discrete abelian group and $f \in \ell^{\infty}(G) \backslash C_{o}(G)$, then $\{\gamma f: \gamma \in E\}$ is not relatively weakly compact if $E \subset \widehat{G}$ has non-empty interior. That result will follow from an existence theorem for $I_{o}$-sets, as follows.

iii) Every infinite subset of a discrete abelian group $\Gamma$ contains an infinite $I_{o}$ set such that for every neighbourhood $U$ of the identity of $\widehat{\Gamma}$ the interpolation (except at a finite subset depending on $U$ ) can be done using at most 4 point masses.

iv) A new proof that $B(G) \subset W A P(G)$ for abelian groups is given that identifies the weak limits of translates of Fourier-Stieltjes transforms.

v) Analogous results for $C_{o}(G), A_{p}(G)$, and $M_{p}(G)$ are given.

vi) Semigroup compactifications of groups are studied, both abelian and non-abelian: the weak* closure of $\widehat{G}$ in $L^{\infty}(\mu)$, for abelian $G$; and when $\rho$ is a continuous homomorphism of the locally compact group $\Gamma$ into the unitary elements of a von Neumann algebra $\mathcal{M}$, the weak* closure of $\rho(\Gamma)$ is studied.
\end{abstract}

\section{INTRODUCTION}

Let $G$ be a locally compact abelian group. Let $C B(G)$ denote the bounded continuous functions on $G$. Let $M(G)$ denote the space of bounded regular Borel measures on $G$. We shall denote the Fourier-Stieltjes transform of a measure $\mu$ by $\hat{\mu}$. The symbol "^" will also be applied to sets of measures, e.g, $F^{\Upsilon}$.

This paper is motivated first by the vast body of results on the almost periodic functions $f \in C B(G)$, resp. weakly almost periodic functions, on $G$ : those are the functions such that the orbits $O^{(*)}(f)=\left\{\delta_{x} * f: x \in G\right\}$ are norm compact, resp. weakly compact. A second motivation is the literature on translation results for Fourier-Stieltjes transforms on locally compact abelian groups; for a survey, see, e.g., [15, pp. 234-238] or [4].

We study variations on these as follows: we replace translation by elements $x \in G$ with multiplication by elements of $\Gamma$, or we replace $C B(G)$ with other Banach spaces

Received by the editors January 23, 2003 and, in revised form, December 8, 2004.

2000 Mathematics Subject Classification. Primary 43A15, 43A10; Secondary 46L10.

Key words and phrases. Almost periodic functions, $I_{o}$-sets, locally compact abelian groups, $p$-multipliers, weak closure of the characters, weak and weak* closures of translates, weakly almost periodic functions.

Both authors were partially supported by NSERC grants. 
related to $G$, or we do both. In all cases, we ask if the orbit is relatively weakly compact, or whether the orbit has relatively weakly compact subsets.

An interesting theory in the context of a translational module over a locally compact abelian group $G$, which is, among other things, a locally convex space of measures, has been developed by Argabright and Gil de Lamadrid in [1]. An almost periodic measure is defined in [1] as one for which the set of translates has compact closure.

For results related to some of ours, see Host, Méla and Parreau [25].

Our notation will be

$$
\begin{aligned}
O^{(\times)}(S) & =\{\gamma \cdot S: \gamma \in \Gamma\} \text { and } \\
O^{(*)}(S) & =\left\{L_{g} S: g \in G\right\},
\end{aligned}
$$

where $L_{g} S$ is the left translation of $S$ by $g$, defined appropriately depending on whether $S$ is a function, a measure, or an element of some other space on $G$ closed under left translations. If $\Lambda$ (resp. $E$ ) is a subset of $\Gamma$ (resp. $G$ ), we define

$$
\begin{aligned}
O_{\Lambda}^{(\times)}(S) & =\{\gamma \cdot S: \gamma \in \Lambda\} \text { and } \\
O_{E}^{(*)}(S) & =\left\{L_{g} S: g \in E\right\} .
\end{aligned}
$$

Thus, we ask whether the restricted sets of translates $O_{\Lambda}^{(\times)}(S)$ and $O_{E}^{(*)}(S)$ are relatively compact in the weak (or other) topology in their containing space. For example, see Theorem 4.1(2) and Theorem 4.5.

We also consider analogues of those results for non-abelian groups, and some compactifications of locally compact groups. We have tried to be thorough in our examination of the effect translation and multiplication by characters has on spaces connected with locally compact groups: $C_{o}(G), L^{1}(G), A_{p}(G), M_{p}(G)$, and $L^{\infty}(G)$.

To give more detail, this paper is organized as follows. Some preliminaries (many measure theoretic) are given in Section 2 . In Section 4 we consider orbits of the form

$$
O^{(\times)}(f)=\{\gamma f: \gamma \in \Gamma\} \subset L^{\infty}(\mu),
$$

where $\mu \in M(G)$ and $f \in L^{\infty}(\mu)$. The section's main result is that $O^{(\times)}(f)$ is weakly compact (in the $\sigma\left(L^{\infty}(\mu), L^{\infty}(\mu)^{*}\right)$ topology) only if $f=0$ or $\mu$ is discrete. We also give a new proof of Theorem 3.1 of [27, as well as a stronger version of that result: if $G$ is discrete and $\Lambda \subset \Gamma$ has non-empty interior and if $f \in L^{\infty}(G) \backslash c_{o}(G)$, then the orbit $O_{\Lambda}^{(\times)}(f)=\{\gamma f: \gamma \in \Lambda\}$ is not relatively weakly compact in $L^{\infty}(G)$. That proof depends on an interpolation theorem for $I_{o}$-sets, which we prove in Section 3 .

In Section 5 we consider orbits using elements of $M(G)$. We give a new proof of the fact that Fourier-Stieltjes transforms are weakly almost periodic, characterizing nets of translates that converge weakly: $\delta_{\gamma_{\alpha}} *(\hat{\mu})$ converges weakly in $C B(\Gamma)$ iff $\gamma_{\alpha}$ converges weak* in $L^{\infty}(\mu)$ (Theorem [5.1). We give related results.

Section 6 considers orbits arising from elements of $A_{p}(G)$ and the $L_{p}$-multipliers on $G$.

In Section 7 we consider $f \in L^{1}(G)$, examining orbits of the forms (1.3)-(1.4).

In Section 8 we consider orbits arising from $f \in C_{o}(G)$.

In Sections 910 we look at compactifications of $\Gamma$, using some results from Sections 2 4. Section 9 relates properties of the semigroup $\overline{\Gamma(\mu)}$ to properties of $\mu$, 
and Section 10 examines implications of the condition

$$
\limsup _{\gamma \rightarrow \infty}|\hat{\mu}(\gamma)|<1
$$

for probablity measures (such a measure $\mu$ is is called "full"). The opposite $\oint^{1}$ condition, of a measure being "Dirichlet" is of interest in Section 4:

$$
\limsup _{\gamma \rightarrow \infty} \operatorname{Re} \hat{\mu}(\gamma)=1 \text {. }
$$

A set $E \subset G$ is a "Dirichlet set" if there exists a sequence of continuous characters $\gamma_{j} \rightarrow \infty$ such that $\gamma_{j} \rightarrow 1$ uniformly on $E$.

In Section 11 we consider mappings of a (possibly non-abelian) group $G$ into the unit ball of a von Neumann algebra by a *-homeomorphism.

We conclude with some open problems.

\section{Preliminaries}

Remark 2.1. Our basic technique in deciding whether a set is relatively weakly compact or not is to use the fact that if $X$ is relatively weakly compact, then the weak and weak* topologies agree on the weak closure of $X$. (That is because the topologies are Hausdorff, and the closure of $X$ is compact in the stronger, that is, the weak-topology.) Generally, we find that 0 is in the weak* closure of $X$ but not in the weak closure. Also, we usually show that 0 is not in the weak closure of $X$ by using a multiplicative linear functional on the algebra of which $X$ is a subset.

To illustrate the point in a particularly simple way, we prove the (known) fact that the charisteric function of non-negative integers, $H=\chi_{[0, \infty)}$, is not weakly almost periodic in $L^{\infty}(\mathbb{Z})$. We obtain a slightly stronger result, namely, that if a subset of $\left\{\delta_{n} * H: n \in \mathbb{Z}\right\}$ is relatively weakly compact, then it is finite. Indeed, let $\Xi_{+} \in L^{\infty}(\mathbb{Z})^{*}$ be any accumulation point of $\delta_{n}$, as $n \rightarrow \infty$, and let $\Xi_{-} \in L^{\infty}(\mathbb{Z})^{*}$ be any accumulation point of $\delta_{n}$, as $n \rightarrow-\infty$. Then

$$
\begin{aligned}
& \left\langle\Xi_{+}, \delta_{m} * H\right\rangle=1 \text { and }\left\langle\Xi_{-}, \delta_{m} * H\right\rangle=0 \forall m \in \mathbb{Z}, \\
& \delta_{m} * H \rightarrow 0 \text { weak* as } m \rightarrow \infty \text { and } \\
& \quad \delta_{m} * H \rightarrow \mathbf{1} \text { weak }^{*} \text { as } m \rightarrow-\infty .
\end{aligned}
$$

If $E \subset[0, \infty)$, and $O_{E}^{(*)}(H)=\left\{\delta_{m} * H: m \in E\right\}$ were relatively weakly compact, then the weak* and weak topologies would agree on the weak closure $\overline{O_{E}^{(*)}(H)}$ of $O_{E}^{(*)}(H)$, so $0 \in \overline{O_{E}^{(*)}(H)}$ by (2.2), contradicting (2.1). A similar argument applies when $E \subset(-\infty, 0]$, using (2.3) in place of (2.2).

An important tool of this paper is the following lemma, which we use to show that 0 belongs to weak* closures. We will give a continuous measure version of this idea, which might be thought of as a singular measure version of the Riemann-Lebesgue Lemma.

Lemma 2.2. Let $\mu$ be a continuous measure on the locally compact abelian group $G$. Let $\Gamma$ be the dual group of $G$. Then $0 \in \overline{\Gamma(\mu)}$, the weak* closure of $\Gamma$ in $L^{\infty}(\mu)$.

\footnotetext{
${ }^{1}$ It is easy to show that if $\mu$ is a probability measure, then $\lim \sup _{\gamma \rightarrow \infty} \operatorname{Re} \hat{\mu}(\gamma)=1$ if and only if $\lim \sup _{\gamma \rightarrow \infty}|\hat{\mu}(\gamma)|=1$.
} 
Proof. It will suffice to show that if $k \geq 1$ and $\mu_{1}, \ldots, \mu_{k} \ll \mu$, then there exists $\gamma_{\ell} \in \Gamma$ such that

$$
\lim _{\ell \rightarrow \infty} \hat{\mu}_{j}\left(\gamma_{\ell}\right)=0 \text { for } 1 \leq j \leq k
$$

With this in mind, we let $\left\{V_{\alpha}\right\}$ be a neighbourhood basis of relatively compact sets at $0 \in G$. Under the relation of inclusion, $\left\{V_{\alpha}\right\}$ is a directed set. For each $\alpha$, let $f_{\alpha}$ be a continuous positive-definite function on $G$ such that $\operatorname{Supp} f_{\alpha} \subset V_{\alpha}$ and $f_{\alpha}(0)=1$, where 0 is the identity in $L^{1}(G)$ for all $\alpha$. That is,

$$
\hat{f}_{\alpha} \geq 0, \text { and } \int_{\Gamma} \hat{f}_{\alpha} d \gamma=1
$$

Then, by a result of Eberlein [10] (or see [32, 5.6.9]),

$$
\lim _{\alpha} \int_{\Gamma} \hat{f}_{\alpha}|\hat{\mu}|^{2} d \gamma=\sum_{x \in G}|\mu(\{x\})|^{2} .
$$

Since $\mu$ is continuous, the right-hand side of (2.6) is zero.

Then (2.5)-(2.6) and $|\hat{\mu}|^{2} \geq 0$ imply that $\hat{\mu}$ cannot be bounded away from zero, that is, 0 must belong to the closure of $\hat{\mu}(\Gamma)$.

Now let $\mu_{1}, \ldots, \mu_{k} \ll \mu$. Replacing $\mu$ in (2.6) with $\mu=\sum_{j} \mu_{j} * \tilde{\mu}_{j}$, we see that $\lim _{\alpha} \int \hat{f}_{\alpha}\left(\sum_{j}\left|\hat{\mu}_{j}\right|^{2}\right)^{2} d \gamma=0$. Hence (2.4) must hold. The conclusion now follows.

We now give an abstract version of the previous lemma.

Assumptions 2.3. Let $\mu$ be a finite positive regular Borel measure on the locally compact Hausdorff space $X$. We let $S^{*}(X, \mu)$ denote the elements of $L^{\infty}(\mu)$ that have absolute value one $\mu$-almost everywhere. Let $\rho: \Gamma \rightarrow S^{*}(X, \mu)$ be a group homomorphism from a locally compact abelian group $\Gamma$ such that $\rho(\gamma) \in C B(X)$ for all $\gamma$ and such that $\rho(\Gamma)$ separates the points of $X$.

Corollary 2.4. Suppose that under Assumptions 2.3 , the measure $\mu$ is a continuous measure. Then 0 is a weak ${ }^{*}$ accumulation point of a net in $O^{(\times)}(f)=\{\rho(\gamma): \gamma \in$ $\Gamma\}$.

Proof. Let $G^{\prime}$ be the dual group of $\rho(\Gamma)$ when the latter is given the discrete topology. Since $\rho(\Gamma)$ separates points of $X, X$ is mapped homeomorphically into $G^{\prime}$ and, therefore, the image of $\mu$ is a continuous measure on $G^{\prime}$. Because $\mu$ is a continuous measure, considered as a measure on $G^{\prime}$, we may apply Lemma 2.2 to conclude that $0 \in \overline{\rho(\Gamma)}$ (the weak* closure).

When weak limits are considered, we do not have the conclusion of Corollary 2.4.

Lemma 2.5. Under Assumptions 2.3, let $F$ be any weak limit of a net in $O(f)=$ $\{\rho(\gamma): \gamma \in \Gamma\}$. Then $F$ has absolute value one everywhere as an element of $L^{\infty}(\mu)^{* *}$.

Proof. Fix a point $w$ in the support of $\mu$, considered as a measure on $\Delta\left(L^{\infty}(\mu)\right)$. Then $\left|\rho\left(\gamma_{\alpha}\right)(w)\right|=1$ for all $\alpha$, since evaluation of $\rho\left(\gamma_{\alpha}\right)$ at $w$ is given by the bounded linear functional

$$
\rho\left(\gamma_{\alpha}\right) \mapsto \int \rho\left(\gamma_{\alpha}\right) d \delta_{w}=\left\langle\rho\left(\gamma_{\alpha}\right), \delta_{w}\right\rangle
$$


which maps $L^{\infty}(\mu) \rightarrow \mathbb{C}$. In (2.7), the integration is against the unit point mass at $w \in \Delta\left(L^{\infty}(\mu)\right)$, and we are identifying elements of $L^{\infty}(\mu)$ with their Gel'fand transforms. Hence, the weak limit $F$ of elements of $\rho(\Gamma)$ has $|F|=1$ as an element of $L^{\infty}(\mu)^{* *}$.

Convergence in $S^{*}(X, \mu)$. The following is well known (e.g., [24, pp. 19-20] for some of the equivalences below), but it will be useful to have a statement here.

Lemma 2.6. Let $\mu$ be a probability measure on the measurable space $(X, \Sigma)$. Let $\Gamma=S^{*}(X, \mu)=\left\{f \in L^{\infty}(\mu):|f| \equiv 1\right.$ a.e. $\left.d \mu\right\}$. Let $\left\{\gamma_{\alpha}\right\}$ be a net in $\Gamma$ and $\gamma \in \Gamma$. Then the following are equivalent:

(1) $\gamma_{\alpha} \rightarrow \gamma$ weak ${ }^{*}$, that is, in the $\sigma\left(L^{\infty}(\mu), L^{1}(\mu)\right)$ topology.

(2) $\gamma_{\alpha} \rightarrow \gamma$ in $L^{2}(\mu)$-norm.

(3) $\gamma_{\alpha} \rightarrow \gamma$ in $L^{1}(\mu)$-norm.

(4) For some $1<p<\infty, \gamma_{\alpha} \rightarrow \gamma$ in $L^{p}(\mu)$-norm.

Remark 2.7. Let $\rho, \Gamma$, and $F$ be as in Lemma 2.5. Then $F$ is a weak* limit of elements of $O(f)=\{\rho(\gamma): \gamma \in \Gamma\}$. This is immediate from the fact that the weak topology is stronger than the weak* topology.

Proof of Lemma 2.6. (1) $\Rightarrow(2) . \int\left|\gamma-\gamma_{\alpha}\right|^{2} d \mu=2-2 \operatorname{Re} \int \gamma_{\alpha} \bar{\gamma} d \mu$. Since $\gamma \in L^{1}(\mu)$, weak* convergence in $L^{\infty}(\mu)$ implies $\int \gamma_{\alpha} \bar{\gamma} d \mu \rightarrow \int|\gamma|^{2} d \mu=1$, and (2) follows.

$(2) \Rightarrow(3)$ is immediate from the Cauchy-Schwarz-Bunyakowski inequality:

$$
\int\left|\gamma-\gamma_{\alpha}\right| d \mu \leq\left(\int\left|\gamma-\gamma_{\alpha}\right|^{2} d \mu\right)^{1 / 2}(1)^{1 / 2}
$$

$(3) \Rightarrow(4)$. Let $1<p<\infty$. Then, since the $\gamma$ 's have modulus one,

$$
\begin{aligned}
\int\left|\gamma-\gamma_{\alpha}\right|^{p} d \mu & =\int\left|\gamma-\gamma_{\alpha}\right|\left|\gamma-\gamma_{\alpha}\right|^{p-1} d \mu \\
& \leq 2^{p-1} \int\left|\gamma-\gamma_{\alpha}\right| d \mu .
\end{aligned}
$$

(4) $\Rightarrow(1)$. Convergence in $L^{p}$-norm implies that $\int F\left(\gamma-\gamma_{\alpha}\right) d \mu \rightarrow 0$ for each $F \in L^{p^{\prime}}(\mu)$, in particular, when $F$ is a (bounded) measurable step function. Let $f \in L^{1}(\mu)$ and $\epsilon>0$. Then there is a (bounded) step function $F$ such that $\|f-F\|_{L^{1}(\mu)}<\epsilon$. Then

$$
\int f\left(\gamma-\gamma_{\alpha}\right) d \mu=\int(f-F)\left(\gamma-\gamma_{\alpha}\right) d \mu+\int F\left(\gamma-\gamma_{\alpha}\right) d \mu .
$$

The first term on the right of (2.8) is bounded by $2 \epsilon$ (just separate into two terms and use the fact that the $\gamma \mathrm{s}$ have modulus one). The second term tends to 0 by weak convergence in $L^{p}$. Hence,

$$
\underset{\alpha}{\limsup } \mid \int f\left(\left(\gamma-\gamma_{\alpha}\right) d \mu \mid<3 \epsilon\right.
$$

so $(4) \Rightarrow(1)$.

Corollary 2.8. Let $\mu$ be a probability measure on the measurable space $(X, \Sigma)$. Then the multiplication in $S^{*}(X, \mu)$ is continuous in both variables simultaneously under any of the topologies of Lemma 2.6(1)-(4) and in the weak topology. 
Proof. [24, p. 19] We begin with the four topologies of Lemma 2.6. Let $\gamma_{\alpha} \rightarrow \gamma$ and $\lambda_{\beta} \rightarrow \lambda$ in any one of those first four topologies, and, hence, in all of them, in particular in the $L^{1}$ norm topology. Then

$$
\left|\gamma_{\alpha} \bar{\lambda}_{\beta}-\gamma \bar{\lambda}\right| \leq\left|\gamma_{\alpha}\left(\bar{\lambda}_{\beta}-\bar{\lambda}\right)\right|+\left|\left(\gamma_{\alpha}-\gamma\right) \bar{\lambda}\right|=\left|\lambda_{\beta}-\lambda\right|+\left|\gamma_{\alpha}-\gamma\right| .
$$

We apply Lemma 2.6 and (2.9) to conclude that the $L^{1}$-convergence of the $\gamma_{\alpha}$ and $\lambda_{\beta}$ imply that $\gamma_{\alpha} \bar{\lambda}_{\beta}$ converges in $L^{1}(\mu)$ norm to $\gamma \bar{\lambda}$. Another application of Lemma 2.6 shows that $\gamma_{\alpha} \bar{\lambda}_{\beta}$ converges to $\gamma \bar{\lambda}$ in the other topologies, except for the weak topology.

Now assume that $\gamma_{\alpha} \rightarrow \gamma$ and $\lambda_{\beta} \rightarrow \lambda$ weakly. Then Lemma 2.6(1) $\Leftrightarrow$ (3) shows that $\gamma_{\alpha} \rightarrow \gamma$ and $\lambda_{\beta} \rightarrow \lambda$ in $L^{1}(\omega)$-norm, for every $\omega \in L^{\infty}(\mu)^{*}$. (We work in $L^{\infty}(\omega)$, instead of $L^{\infty}(\mu)$.) Now the proof for the preceding case shows that $\gamma_{\alpha} \bar{\lambda}_{\beta}$ converges to $\gamma \bar{\lambda}$ in $L^{1}(\omega)$-norm to $\gamma \bar{\lambda}$, and, hence, in the weak topology.

\section{An interpolation theOREM ON $I_{o}$-SETS}

In this section we shall establish an interpolation theorem for $I_{o}$-sets (defined below) that we need to prove the main result, Theorem 4.5, in Section 4 .

Before stating the theorem, some background information may be useful. A subset $\Lambda$ of the discrete abelian group $\Gamma$ is an " $I_{o}$-set" iff every bounded function on $\Lambda$ can be extended to an almost periodic function on $\Gamma$. This is equivalent to having every bounded function on $\Lambda$ being the Fourier-Stieltjes transform of a discrete measure. See [28, p.165ff] for references. The set $\Lambda$ is a "Sidon set" if every bounded function on $\Lambda$ can be extended to a Fourier-Stieltjes transform on all of $\Gamma$. Let $G$ be the dual group of $\Gamma$ and $K \subset G$. We say that $K$ is "associated with" $\Lambda$ if there exists a constant $C>0$ and a finite subset $F \subset \Lambda$ that for all trigonometric polynomials $p(x)$ on $G$ with coefficients from $\Lambda \backslash F, \sum_{x \in G}\left|p(x) \leq C \sup _{x \in K}\right| p(x) \mid$. If $G$ is compact and connected, then every Sidon set in $\Gamma$ is associated with every $K \subset G$ with int $K \neq \emptyset$ by results of Déchamps-Gondim [8]. The result of $[8$ is stronger that we state. See 8 and also [28, p. 109ff] for details and a still stronger result.

It will be apparent from the preceding paragraph that Theorem 3.1 is a variant of [8]. The papers [8], 29], and [30], suggested Theorem [3.1, A better result may be possible. We have stated and proved what would give us Theorem 4.5 .

Theorem 3.1. Let $G$ be a discrete abelian group with dual group $\Gamma$. Let $H \subset G$ be infinite. Then there exists an infinite subset $H^{\prime} \subset H$ and integer $q \geq 2$ such that for every open neighbourhood $U$ of the identity in $\Gamma$, and every $\epsilon>0$, there exists a finite subset $F \subset H^{\prime}$ such that for every complex valued function $\phi: H^{\prime} \rightarrow \mathbb{C}$ with

$$
\phi^{q} \equiv 1
$$

everywhere on $H^{\prime}$, there exists $x \in U$ with

$$
|\langle x, \lambda\rangle-\phi(\lambda)| \leq \epsilon \text { for } \lambda \in H^{\prime} \backslash F
$$

The integer $q$ is not necesarily the order of $\Gamma$; it may be a prime factor of the order of $\Gamma$; see, e.g., Corollary 3.10 below.

The following corollary is immediate from Theorem 3.1, and was previously obtained by Hartman and Ryll-Nardzewski [17, Kalton [16, Galindo and Hernández [13, and Kunen and Rudin [26]. 
Corollary 3.2. Let $G$ be a discrete abelian group and $H$ an infinite subset of $G$. Then $H$ contains an infinite subset that is an $I_{o}$-set.

3.0.1. Outline of the proof of Theorem 3.1. We begin the proof of Theorem 3.1 by consideration of particular cases $G$, starting with $G=\mathbb{Z}$. We then show that if Theorem 3.1 holds for a subgroup or a quotient group of $\Gamma$, it holds for $\Gamma$. After some other reductions, we consider the case that $H$ contains an infinite independent set. The case that $H$ has an infinite subset of elements of the same finite order is reduced to the case of an infinite independent set. We then prove the theorem for the general case.

Lemma 3.3. Assume the hypotheses of Theorem 3.1. Suppose that $G=\mathbb{Z}$. Then the conclusion of Theorem 3.1 holds.

Proof. The proof proceeds from the observation that for each interval $(a, b) \subset \mathbb{T}$ and $\lambda \in \mathbb{Z}, \lambda \neq 0$, the range of $x \rightarrow e^{2 \pi i \lambda x} x \in(a, b)$ spreads out to cover all of $\mathbb{T}$ as $\lambda \rightarrow \infty$. It is then easy to construct, for each $\phi$, a convergent sequence in $(a, b)$ which yields the required element. Here are the details. Let a non-empty interval $(a, b)=\left(a_{1}, b_{1}\right) \subset \mathbb{T}$ be given such that $\left[a_{1}, b_{1}\right] \subset U$ (using closure here will ensure that our limit point is in $U)$. Choose $\lambda_{1} \in H$ such that

$$
\left\{e^{2 \pi i \lambda_{1} x} ; x \in\left(a_{1}, b_{1}\right)\right\}=\mathbb{T} .
$$

Choose $\lambda_{2}>\lambda_{1}, \lambda_{2} \in H$ so large that for any interval $I \subset \mathbb{T}$ of length at least $\frac{b_{1}-a_{1}}{400 \pi \lambda_{1}}$

$$
\left\{e^{2 \pi i \lambda_{2} x}: x \in I\right\}=\mathbb{T} .
$$

Proceeding in this way, we find a strictly increasing sequence $\lambda_{j} \rightarrow \infty$ such that for $j \geq 2,\left\{e^{2 \pi i \lambda_{j} x} ; x \in I\right\}=\mathbb{T}$ whenever $I$ has length at least $\frac{\left(b_{1}-a_{1}\right)}{400 \pi \prod_{k=1}^{j-1} \lambda_{k}}$. Let $H^{\prime}=\left\{\lambda_{j}: j \geq 1\right\}$. Let $\phi: H^{\prime} \rightarrow \mathbb{T}$ be given. By (3.4), there exists $x_{1} \in\left(a_{1}, b_{1}\right)$ with $e^{2 \pi i \lambda_{1} x_{1}}=\phi\left(\lambda_{1}\right)$. Then

$$
\left|x-x_{1}\right|<\frac{1}{200 \pi \lambda_{1}} \Rightarrow\left|e^{2 \pi i \lambda_{1}, x}-\phi\left(\lambda_{1}\right)\right|<1 / 100 .
$$

Let $\left(a_{2}, b_{2}\right)=\left(x_{1}-400 \pi / \lambda_{1}, x_{1}+400 \pi / \lambda_{1}\right)$. By (3.4),

$$
\left\{e^{2 \pi i \lambda_{j} x} ; x \in\left(a_{2}, b_{2}\right)\right\}=\mathbb{T} \text { for } j \geq 2 .
$$

Therefore, there exists $x_{2} \in\left(a_{2}, b_{2}\right)$ such that $e^{2 \pi i \lambda_{2} x_{2}}=\phi\left(\lambda_{2}\right)$. Then

$$
\left|x-x_{2}\right|<1 /\left(200 \pi \lambda_{1} \lambda_{2}\right) \Rightarrow\left|e^{2 \pi i \lambda_{2}, x}-\phi\left(\lambda_{2}\right)\right|<1 / 100 .
$$

Let $\left(a_{3}, b_{3}\right)=\left(x_{2}-\left(400 \pi / \lambda_{1} \lambda_{2}\right), x_{2}+\left(400 \pi / \lambda_{1} \lambda_{2}\right)\right)$.

Proceeding in this way, we find a strictly decreasing sequence of intervals $\left(a_{j}, b_{j}\right)$ and elements $x_{j} \in\left(a_{j}, b_{j}\right)$ with

$$
e^{2 \pi i \lambda_{j} x_{j}}=\phi\left(\lambda_{j}\right) \text { and }\left|e^{2 \pi i \lambda_{j}, x}-\phi\left(\lambda_{j}\right)\right|<1 / 100 \text { if } x \in\left(a_{j}, b_{j}\right) .
$$

Let $x_{o}=\lim x_{j}$. Then $\left|e^{2 \pi i \lambda_{j} x_{o}}-\phi\left(\lambda_{j}\right)\right| \leq 1 / 100$ for all $j \geq 1$. That completes the proof of Lemma 3.3 .

Lemma 3.4 (Subgroup Lemma). Assume the hypotheses of Theorem 3.1. Let $\Xi$ be a subgroup of $G$. Suppose $H \cap \Xi$ is infinite. Suppose also that the conclusion of Theorem 3.1 holds for $\Xi$ and $H \cap \Xi$. Then the conclusion of Theorem 3.1 holds for $G$. 
Proof. Let $L=\Xi^{\perp}$. Let $q$ and $H^{\prime}$ be given for $H \cap \Xi$, and let $q$ and $H^{\prime} \subset H \cap \Xi$ be given by Theorem 3.1 applied to $\Xi$ and $H \cap \Xi$. Let $U \subset G$ be an open neighbourhood of the identity of $\Gamma$, and let $U_{1}$ be the image of $U$ under the quotient homorphism $G \rightarrow G / H$. Then $U_{1}$ is an open neighbourhood of the identity of $G / H$, since the quotient mapping is open. Hence, there exists a finite set $F \subset H^{\prime}$ and a representative $x+H \in U_{1}$ such that (3.2) holds for all $\lambda \in H^{\prime} \backslash F$, that is, $|\langle x+H, \lambda\rangle-\phi(\lambda)|<\epsilon$ for all $\lambda \in H^{\prime} \backslash F$ and all $\phi: H^{\prime} \rightarrow \mathbb{T}$ with $\phi^{q}=1$. Since $L=\Xi^{\perp}$, we can replace $x+L$ with its representative, so that $|\langle x, \lambda\rangle-\phi(\lambda)|<\epsilon$ for all $\lambda \in H^{\prime} \backslash F$. That establishes Theorem 3.1 for $G$.

The following corollaries are (nearly) immediate.

Corollary 3.5. Assume the hypotheses of Theorem 3.1. Without loss of generality in the proof of Theorem 3.1, we may assume that $H$ is countable and generates $G$.

Corollary 3.6. Assume the hypotheses of Theorem 3.1. Suppose that $H$ contains an infinite number of elements of a singly generated subgroup of $G$. Then the conclusion of Theorem 3.1 holds for $G$ and $H$.

Proof. Indeed, we can identify the singly generated subgroup of $G$ with $\mathbb{Z}$. Now Corollary 3.6] follows from Lemma 3.3 and Subgroup Lemma 3.4 .

Lemma 3.7 (Quotient Group Lemma). Assume the hypotheses of Theorem 3.1, Let $\Xi$ be a subgroup of $G$. Suppose that the image $H+\Xi$ in $G / \Xi$ is infinite. Suppose also that the conclusion of Theorem 3.1 holds for $H+\Xi$ and $G / \Xi$. Then the conclusion of Theorem 3.1 holds for $G$.

Proof. Let $L=\Xi^{\perp}$. Then $U$ is replaced with the intersection $U \cap L$, which is a relatively open neighbourhoood of the identity of $L$. Let $H_{1} \subset H$ be such that $H_{1}+\Xi=H+\Xi$ and the mapping from $H_{1} \rightarrow H_{1}+\Xi$ is one-to-one 2 Define $\phi^{\prime}: H_{1}+\Xi \rightarrow \mathbb{C}$ by $\phi^{\prime}(\lambda+\Xi)=\phi(\lambda)$. Let $q$ be given for $G / \Xi$. By the hypotheses of this lemma, there exists $H^{\prime} \subset H_{1}$ such that if $\phi: H \rightarrow \mathbb{C}$ satisfies (3.1), then there exists $x \in U \cap L$ with $|\langle x, \lambda\rangle-\phi(x)|<\epsilon$, by (3.2) applied to $G / \Xi$. Since $L=\Xi^{\perp},\langle x, \lambda\rangle=\langle x, \lambda+\Xi\rangle$ for all $\lambda \in H^{\prime}$, so the conclusion of Theorem 3.1 holds for $G$ and $H$.

Corollary 3.8. Assume the hypotheses of Theorem 3.1. Suppose that $H$ contains an infinite number of elements of a coset of a finitely generated subgroup of $G$. Then the conclusion of Theorem 3.1 holds for $G$ and $H$.

Proof. Suppose that $H$ contains an infinite set that is contained in a coset of a finitely generated subgroup of $G$. We apply the Subgroup Lemma 3.4, so that we may assume that $G$ is finitely generated by the generators of the finitely generated subgroup plus a representative of the coset. Then we can find one of the generators, say $\omega$, of (our now finitely generated) $G$ such that $\omega$ has infinite order and the projection (a quotient mapping!) of $H$ onto the subgroup generated by $\omega$ is infinite. We apply the Quotient Group Lemma, Lemma 3.7 and Lemma 3.3 to conclude that the conclusion of Theorem 3.1 holds for $G$ and $H$.

Lemma 3.9. Assume the hypotheses of Theorem 3.1. Suppose that $H$ contains an infinite independent set. Then the conclusion of Theorem 3.1 holds for $G$ and $H$.

${ }^{2}$ If we apply Subgroup Lemma 3.4 first, we can reduce to the case of countable $G$ and avoid the use of the Axiom of Choice in the construction of $H_{1}$. 
Proof. Suppose that $H$ contains a countable infinite independent set $H^{\prime}$. If all the elements of $H^{\prime}$ have the same order $q \geq 2$, then that $q$ will be the $q$ of the assertion of Theorem 3.1. Otherwise, we will take $q=2$ and assume that the order of every element of $H^{\prime}$ is greater than $100 / \epsilon$. Let $L$ be the subgroup of $G$ generated by $H^{\prime}$. By Subgroup Lemma 3.4 we may assume that $G=L$. We consider $L$ : it is a direct sum of cyclic groups, and $\widehat{L}$ is the direct product of the compact dual groups of those cyclic groups. But now the conclusion will follow easily from the structure of product groups: we define $x$ by its coordinates, chosing the $j^{\text {th }}$ coordinate $x_{j}$ so that $\left|\left\langle x_{j}, \lambda_{j}\right\rangle-\phi\left(\lambda_{j}\right)\right|<\epsilon$. Here, we will have equality if $q<\infty$. Since we have a product group, $\left\langle x_{j}, \lambda_{k}\right\rangle=1$ if $j \neq k$. Then $x=\prod x_{j}$ will do, where we take a finite number of the $x_{j}=1$, so that the resulting product is indeed in $U$. The finite set $F$ will thus depend on the neighbourhood $U$ : the smaller $U$ is, the larger $F$ is. Of course, $U$ can be taken as a product set of the form $\{0\} \times \cdots \times\{0\} \times \prod_{\ell=1}^{\infty} \mathbb{Z}_{q}$, and then $F$ is the set of elements of $H$ which are non-zero in the factors of $E$ which are non-zero. Therefore, the conclusion of Theorem 3.1 holds for $G$ and $H$.

Corollary 3.10. Assume the hypotheses of Theorem 3.1. Suppose that $H$ contains an infinite set of elements all having the same finite order $q$. Then the conclusion of Theorem 3.1 holds for $G$ and $H$.

Proof. Suppose that an infinite set of elements of $H$ had the same finite order $q \geq 2$. First suppose that $q$ is prime. We then claim that $H$ contains an infinite independent set. Indeed, suppose that $I=\left\{\lambda_{1}, \ldots, \lambda_{n}\right\}$ is a maximal independent subset of $H$. If $\lambda \in H \backslash I$, then the maximality of $I$ means that there exist integers $1 \leq a<q$ and $0 \leq a_{j}<q$ with at least one $a_{j}$ not zero such that $a \lambda=\sum_{j} a_{j} \lambda_{j}$. Since the $\lambda_{j}$ are independent and $q$ is prime, we have $m a \lambda=\sum_{j} m a_{j} \lambda_{j}=0$ if and only if $m a_{j} \equiv 0(\bmod q)$ for all $j$, that is, $m=q$. In other words, $\lambda$ has order $q a$, a contradiction, unless $a=1$. Of course, only a finite number $\left(q^{n}\right)$ of elements of $H \backslash I$ can have the form $\lambda=\sum_{j} a_{j} \lambda_{j}$. Eliminating those, we find an independent subset of $H$ that has $n+1$ elements, so $I$ is not maximal. This contradiction establishes the claim, so $H$ does contain an infinite independent set, and therefore, the conclusion of Theorem 3.1 holds for $G$ and $H$.

We now reduce to the subcase that $q$ is prime, as follows. Let $p$ be a prime factor of $q$, and let $q=p r$. Let $\Xi=r G$. Then the image $H+\Xi$ in $G / \Xi$ contains an infinite set of elements of prime order $p$. Hence, the conclusion of Theorem 3.1 holds for $G / \Xi$ and $H+\Xi$, by the preceding paragraph. By the Quotient Group Lemma 3.7. the conclusion of Theorem 3.1 holds for $G$ and $H$.

Proof of Theorem 3.1. By Corollary 3.5, we may assume that $H$ is countable and generates $G$. By Lemma 3.9] we may assume that $H$ does not contain an infinite independent set. By Corollary 3.8 we may assume that no infinite subset of $H$ is contained in a finitely generated subgroup of $G$. Also, by Corollary 3.10, we may assume that $H$ does not contain an infinite set of elements having the same finite order.

Therefore, we may assume that $H$ has no infinite independent subset, and that either

(1) all elements of $H$ have different finite orders, all of which are greater than $100 / \epsilon$; or

(2) $H$ has no infinite independent subset, and all elements of $H$ have infinite order. 
For example, in case (1), $G$ could be roots of unity in $\mathbb{T}$, with the discrete topology. In case (2), $G$ could be the rational numbers $\mathbb{Q}$ with the discrete topology. (The assumption in case (1) that the orders are different and all greater than $100 / \epsilon$ follows from an (other) application of the Subgroup Lemma 3.4.)

We will reduce the second case to the first, and then show that in the first case, the conclusion of Theorem 3.1 holds for $G$ and $H$.

Let $I$ be a maximal independent subset of $H$. Of course, $I$ could contain only the element, 1 and anyway, $I$ is finite. Consider $\Xi=G p(I)$. Then $H+\Xi$ is an infinite subset of $G / \Xi$. Indeed, otherwise, $H$ would be contained in a finitely generated subgroup of $G$, contradicting our assumptions at the start of the proof of Theorem 3.1 Thus, we may assume that $H+\Xi$ is an infinite subset of $H / \Xi$.

By the Quotient Group Lemma 3.7, it will suffice to prove Theorem 3.1 for $G / \Xi$ and $H+\Xi$. We claim that the orders of the elements of $H+\Xi$ are finite. Indeed, if some element $\lambda+\Xi$ had infinite order in $G / \Xi$, then $I \cup\{\lambda\}$ would be independent, contradicting the maximality of $I$.

This completes the reduction to case (1).

In this case any $q \geq 2$ will do. For simplicity, we will use

$$
q=2 .
$$

We recall the assumptions that the order of each element of $H$ is at least $10 q / \epsilon$ and that $H$ is countable and generates $G$. Pick any $\lambda_{1} \in H$. Let $I_{1}=\left\{\lambda_{1}\right\}$. Consider $G / G p\left(I_{1}\right)$. Since $G p\left(I_{1}\right)$ is finite, the image $H_{1}=H+G p\left(I_{1}\right) \backslash\{0\}$ is infinite. If $H_{1}$ contained an infinite set of elements of the same finite order, then by the Quotient Group Lemma 3.7, we are done. Hence, we may assume that $H$ contains an element $\lambda_{2}$ such that $\lambda_{2}+G p\left(I_{1}\right)$ has order at least $10 q / \epsilon$ in $G / G p\left(I_{1}\right)$. By induction, we may find a sequence $\lambda_{1}, \lambda_{2}, \ldots$ such that for $I_{k}=\left\{\lambda_{1}, \ldots, \lambda_{k}\right\}$,

$$
\lambda_{k+1}+G p\left(I_{k}\right) \text { has order at least } 10 q / \epsilon \text { in } G / G p\left(I_{k}\right) .
$$

We have not yet completed our selection of $H^{\prime}$, however. We need a further growth condition, which we now describe. Let $F$ be a finite subset of $G$. Suppose $H^{\prime}+G p(F)$ were a finite subset of the quotient group $G / G p(F)$, say

$$
H^{\prime}=\bigcup_{j=1}^{\ell}\left(\lambda_{j}+F\right) .
$$

Then $H^{\prime} \subset G p\left(F \cup\left\{\lambda_{1}, \ldots, \lambda_{\ell}\right\}\right)$, and we are in the situation of Corollary 3.8, which we have eliminated. Thus, we may assume that $F \subset G$ and $\# F<\infty$ implies that

$$
\#\left(H^{\prime}+G p(F)\right)=\infty \text {. }
$$

Similarly, we may assume that $F \subset G$ and $\# F<\infty$ implies the orders of the elements of $H^{\prime}+G p(F)$ are unbounded, and no order repeats infinitely often. Thus, we apply induction to obtain a subset $H^{\prime}=\left\{\lambda_{1}, \lambda_{2}, \ldots\right\} \subset H$, so that for each $n \geq 1$, and $I_{n}=\left\{\lambda_{1}, \ldots, \lambda_{n}\right\}$,

$$
\begin{aligned}
& \#\left(H^{\prime}+G p\left(I_{n}\right)\right)=\infty \text { and } \\
& \text { the order of } \lambda_{n+1}+G p\left(I_{n}\right) \in H^{\prime}+G p\left(I_{n}\right) \text { is at least } 100^{n+1} / \epsilon .
\end{aligned}
$$

Of course, we may still assume that $H^{\prime}$ generates $G$.

Now suppose an open neighbourhood $E$ of $0 \in G$ is given. Since $E$ is open, it contains a basic neighbourhood of the form $U=\left\{x:\left|<\lambda_{j}, x>-1\right|<\delta, j=\right.$ $1, \ldots, m\}$, for some $\delta>0$ and some $m \geq 1$. In fact, we may assume that $U+U \subset E$. 
(This is not the largest neighbourhood basis, but it is a neighbourhood basis at 0 , since we are assuming that $H^{\prime}$ generates $G$.) Let $F=\left\{\lambda_{j}: 1 \leq j \leq m\right\}$ and $\Xi_{m}=G p(F)$.

Now suppose that $\phi: H^{\prime} \rightarrow \mathbb{C}$ satisfying (3.1) is given, that is, $\phi\left(\lambda_{j}\right)= \pm 1$, by (3.6). We must find $x \in E$ such that (3.2) holds. Consider $\lambda_{m+1}$. By (3.10), $\lambda_{m+1}+\Xi_{m}$ has order at least $100^{m} / \epsilon$. Therefore, there exists $x_{m+1} \in \Xi_{m}^{\perp}$ with $\left|\left\langle\lambda_{m+1}, x_{m+1}\right\rangle-\phi\left(\lambda_{m+1}\right)\right|<\epsilon / 2^{m+1}$. Since $x_{m+1} \in \Xi_{m}^{\perp}, x_{m+1} \in U$.

Assume that $k \geq m$. Suppose that for $m \leq n \leq k$,

$$
\begin{aligned}
& \Xi_{n}=G p\left(\left\{\lambda_{1}, \ldots, \lambda_{n}\right\}\right), \\
& x_{n+1} \in \Xi_{n}{ }^{\perp}, \\
& \left|\left\langle\sum_{j=m+1}^{n+1} x_{j}, \lambda_{n+1}\right\rangle-\phi\left(\lambda_{n+1}\right)\right|<\epsilon / 2^{n+1} .
\end{aligned}
$$

From (3.11)- (3.12), $\sum_{n=m+1}^{k} x_{n} \in U$.

Let $\Xi_{k+1}=G p\left(\left\{\lambda_{1}, \ldots, \lambda_{k+1}\right\}\right)$. By (3.10), there exists $x_{k+2} \in \Xi_{k+1}^{\perp}$ such that

$$
\left|\left\langle\sum_{j=m+1}^{k+2} x_{j}, \lambda_{k+2}\right\rangle-\phi\left(\lambda_{k+2}\right)\right|<\epsilon / 2^{k+2} .
$$

Hence, $x_{k+2} \in U$. We let $x$ be any accumulation point of the set of sums $\sum_{j=m+1}^{n+1} x_{j}$, $n \geq m+1$. Straighforward calculations show that $x \in \bar{U} \subset U+U \subset E$. That completes the proof of Theorem 3.1 .

\section{Orbits of $\gamma f$, FOR $f \in L^{\infty}(\mu), \gamma \in \Gamma$, AND $\mu \in M(G)$}

We now give our first variation on the theme of weakly almost periodic functions. It is a rather general version. For the concrete version, just ignore " $\rho(\cdot) . "$

Theorem 4.1. Suppose that under Assumptions 2.3, $\mu$ is continuous and $f \in$ $L^{\infty}(\mu)$ is not the zero function. Then the following hold:

(1) $O(f)=\{\rho(\gamma) f: \gamma \in \Gamma\}$ is not relatively weakly compact in $L^{\infty}(\mu)$.

(2) If $\Lambda \subset \Gamma$ and if $\{\rho(\gamma) f: \gamma \in \rho(\Lambda)\}$ is relatively weakly compact, then every element $\tau$ in the weak ${ }^{*}$ closure of $\rho(\Lambda)$ in $L^{\infty}(\mu)^{* *}$ has

$$
|\tau| f=f \text {, as elements of } L^{\infty}(\mu)^{* *} .
$$

Remarks 4.2. (i) The set $\{\rho(\gamma) f: \gamma \in \Gamma\}$ can have infinite relatively weakly compact subsets (in contrast, e.g., with Remark 2.1 and Proposition 6.2). Here is an example. Suppose $\mu$ is a continuous probability measure on a Dirichlet 3 subset $E$ of $\mathbb{T}$; see (1.7). That means there exist $\gamma_{j} \in \mathbb{Z}$ such that $\gamma_{j} \rightarrow 1$ uniformly on $E$. It follows that $\{f\} \cup\left\{\gamma_{j} f: 1 \leq j<\infty\right\}$ is norm (!) compact.

(ii) Let $G$ be a locally compact abelian group with dual group $\Gamma, \mu \in M(G)$ and $f \in L^{\infty}(\mu)$. Then $\{\gamma f: \gamma \in \Gamma\}$ has weakly compact subsets if and only $f \mu$ is Dirichlet; see Section 10] and 24] for more about Dirichlet measures.

\footnotetext{
${ }^{3}$ Every Kronecker set is a Dirichlet set, and Kronecker sets exist in many groups, including $\mathbb{T}$ 15, 19, 32.
} 
Proof of 4.1. We prove (1) first. We suppose that $O(f)$ is relatively weakly compact and derive a contradiction. The proof is by way of four assertions. (i) We may assume that $f \equiv 1$ a.e. $d \mu$. (ii) 0 is in the weak* closure of $\rho(\Gamma)$. (iii) Relative weak compactness implies that every function in the weak closure of $\rho(\Gamma) f$ is unimodular. (iv) The preceding are contradictory.

(i) Since $f \neq 0$, there exists an element $g \in L^{\infty}(\mu)$ such that $f g$ is a non-zero idempotent in $L^{\infty}(\mu)$.

Of course, if $\{\rho(\gamma) f: \gamma \in \Gamma\}$ were relatively weakly compact, then $\{\rho(\gamma) f g$ : $\gamma \in \Gamma\}$ would also be relatively weakly compact, since multiplication in $L^{\infty}(\mu)$ is separately continuous in the weak topology. Therefore, we may assume that $f$ is idempotent. By replacing $\mu$ by $f \mu$, we see that we may assume that $f \equiv 1$ a.e.

(ii) This is just the conclusion of Corollary 2.4.

(iii) Since $f \equiv 1$, this is exactly the assertion of Lemma 2.5 .

(iv) Now suppose that $\rho(\Gamma)=\{\rho(\gamma): \gamma \in \Gamma\}$ is relatively weakly compact. Let $\overline{\rho(\Gamma)}^{w}$ denote the weak closure of $\rho(\Gamma)$. Then the weak and weak* topologies agree on $\overline{\rho(\Gamma)}^{w}$ by Remark 2.1. Let $\left\{\gamma_{\alpha}\right\}$ be a net in $\rho(\Gamma)$ that converges weak* to 0 . Such a net exists by (ii) above. Then $\left\{\gamma_{\alpha}\right\}$ also converges weakly. But $\left\{\gamma_{\alpha}\right\}$ converges to some element $\gamma$ such that $|\gamma| \equiv 1$ in $L^{\infty}(\mu)$, by (iii). Hence $0=|\gamma|=1$, a contradiction. That completes the proof of (1).

The proof of (2) is a slight elaboration of the preceding. Suppose that there exists $\tau$ in the weak* closure of $\rho(\Lambda)$ such that $|\tau| f \neq f$. As in (i) above, we may assume that $f$ is idempotent and $f \mu=\mu$ (we throw away the part of $f$ where $|\tau| f=f$ ). As in (iii), any weak accumulation point $\tau$ of $\rho(\Lambda)$ must have $|\tau|=1$ as an element of $L^{\infty}(\mu)$. Hence $|\tau| f=f$.

Corollary 4.3. Let $\mu$ be a finite regular Borel positive measure on the locally compact abelian group $G$. If $\mu$ is continuous, then for all $f \in L^{\infty}(\mu) \backslash\{0\}$, the orbit $O^{(\times)}\{\gamma f: \gamma \in \Gamma\}$ is not relatively weakly compact in $L^{\infty}(\mu)$.

Proof. This is almost immediate. The mapping $\rho: \Gamma \rightarrow S^{*}(G, \mu)$ given by $\rho(\gamma)(f)=\gamma f$ separates points of $X$, where $X \subset G$ is the support of $f$.

The mapping is, of course, weak* continuous.

The next corollary is essentially Theorem 3.1 of $[27$.

Corollary 4.4. Let $G$ be a locally compact abelian group with dual group $\Gamma$.

(1) If there exists a non-zero $f \in L^{\infty}(G)$ such that $O^{(\times)}(f)=\{\gamma f: \gamma \in \Gamma\}$ is relatively weakly compact in $L^{\infty}(G)$, then $G$ is discrete.

Suppose that $G$ is discrete. Let $f \in L^{\infty}(G), f \neq 0$. Let $O^{(\times)}(f)=\{\gamma f: \gamma \in \Gamma\}$. Then the following are equivalent:

(2) $f \in c_{o}(G)$.

(3) $O^{(\times)}(f)$ is norm compact.

(4) $O^{(\times)}(f)$ is weakly compact (in the $\sigma\left(L^{\infty}(G), L^{\infty}(G)^{*}\right)$ topology).

Proof. (1) (This argument is very different from that in 27].) Let $U$ be a relatively compact open subset of $G$ such that $f_{\mid U} \neq 0$, and let $\mu$ denote the Haar measure of $G$ restricted to $U$. If $O^{(\times)}(f)$ is relatively weakly compact in $L^{\infty}(G)$, then $\left\{\gamma f \mathbf{1}_{U}: \gamma \in \Gamma\right\}=\mathbf{1}_{U} O^{(\times)}(f)$ would be relatively weakly compact in $L^{\infty}(\mu)$, since 
multiplication in $L^{\infty}(G)$ is weakly continuous in each variable separately. But, by Corollary $4.3, \mathbf{1}_{U} O^{(\times)}(f)$ is not relatively weakly compact in $L^{\infty}(\mu)$ if $\mu$ is not discrete (i.e., iff $G$ is not discrete). This establishes (1).

Theorem 4.5 is a stronger version of the equivalences (2)-(4). That stronger version provides our proof of the equivalences (2)-(4). Our proof is different from [27, Theorem 3.1].

By using a method different from [27, we can obtain a stronger version of Corollary 4.4(2)-(4), as follows.

Theorem 4.5. Let $G$ be a discrete abelian group with dual group $\Gamma$. Let $f \in$ $L^{\infty}(G) \backslash C_{o}(G)$. Let $\Lambda \subset \Gamma$. If Int $\Lambda \neq \emptyset$, then $O_{\Lambda}^{(\times)}(f)=\{\gamma f: \gamma \in \Lambda\}$ is not relatively weakly compact.

Proof of Theorem 4.5. For simplicity, we will write $O_{\Lambda}(f)$ in place of $O_{\Lambda}^{(\times)}(f)$, for this proof.

Because $f(x) \nrightarrow 0$ as $x \rightarrow \infty,|f(x)|>\epsilon$ for some $\epsilon>0$ and an infinite number of $x \in G$. Let $\Lambda$ be that set of $x$. By multiplying $f$ by an element of $L^{\infty}(G)$, we may assume that $f \equiv 1$ on $\Lambda$.

Let $\Lambda \subset \Gamma$ have non-empty interior. We may assume that $\Lambda$ is open. Let $\gamma_{o} \in \Lambda$. Then $O_{\Lambda}(f)$ is relatively weakly compact if and only if $\gamma_{o} O_{\Lambda}(f)$ is relatively weakly compact. Therefore, we may assume that $\Lambda$ is a neighbourhood of the identity.

We now apply Theorem 3.1; let $\Lambda^{\prime}$ and $q$ be given by Theorem 3.1, and let $\Xi$ be any weak* accumulation point of $\left\{\delta_{x}: x \in \Lambda^{\prime}\right\}$ in $L^{\infty}(G)^{*}$. Then every neighbourhood $U$ of the identity of $\Gamma$ contains $\gamma_{U}$ such that

$$
\left|\left\langle\gamma_{U}, x\right\rangle-e^{2 \pi i / q}\right|<1 / 100 \text { except for a finite number of } x \in E^{\prime} .
$$

We then have $\left|\left\langle\Xi, \gamma_{U} f\right\rangle-e^{2 \pi i / q}\right|<1 / 100$, while $\langle\Xi, f\rangle=1$. Hence,

$$
\gamma_{U} f \nrightarrow f \text { weakly as } U \searrow\{0\} \text {, }
$$

though

$$
\gamma_{U} f \rightarrow f \text { weak } *
$$

Of course, if $O_{\Lambda}(f)$ is relatively weakly compact, then $O_{\Lambda}(f)$ would be relatively weak* compact, and the two topologies would agree on $O_{\Lambda}(f)$, which they do not by (4.2) -4.3 .

\section{Orbits IN VARIOUS CONTAINING SPACES OF $\gamma \mu$, FOR $\mu \in M(G)$ AND $\gamma \in \Gamma$}

It has been known since Eberlein's 1949 paper 9 ( or see [5, Cor. 3.4 on p. 37]) that each Fourier-Stieltjes transform $\hat{\mu}$ on a locally compact abelian group is weakly almost periodic, but it does not appear to be equally well known that the elements of the weak closure of the set $O^{(\times)}(\hat{\mu})$ of translates are also Fourier-Stieltjes transforms. In this section, we give a new proof of Eberlein's result, which contains the stronger statement. We then give some applications of our method, and show how an analogous conclusion can be obtained in the non-abelian case. By $B(\Gamma)$, we mean the set of Fourier-Stieltjes transforms on $\Gamma$; by $\frac{d \nu}{d \mu}$ we mean the Radon-Nikodym derivative of $\nu$ with respect to $\mu$. 
Theorem 5.1. Let $\Gamma$ be a locally compact abelian group. Then every FourierStieltjes transform $\hat{\mu}$ is a weakly almost periodic function on $\Gamma$. Furthermore, the following are equivalent:

(1) $\hat{\nu}$ is a weak accumulation point in $L^{\infty}(\Gamma)$ of $\left\{L_{\gamma} \hat{\mu}: \gamma \in \Gamma\right\}$.

(2) $\frac{d \nu}{d \mu}$ is a weak* accumulation point of $\{\gamma: \gamma \in \Gamma\}$ in $L^{\infty}(\mu)$.

In particular, $B(\Gamma)$ is closed under the operation of taking weak limits in $C B(\Gamma)$ of translates of its elements.

Proof. Let $\gamma_{\alpha}$ be any net in $\Gamma$. Without loss of generality, we may assume that $\gamma_{\alpha} \rightarrow f$ weak* $^{*}$ in $L^{\infty}(\mu)$. Let $S \in C B(\Gamma)^{*}$. Then the composition of $S$ with the Fourier-Stieltjes transform (call it $T$, so $\hat{\nu}=T(\nu)$ for all $\hat{\nu} \in B(\Gamma)$ ) is a bounded linear functional on $L^{1}(\mu)$. Hence, there exists an element $F_{S} \in L^{\infty}(\mu)$ such that for all $\nu \ll \mu$,

$$
\langle S, \hat{\nu}\rangle=\langle S, T(\nu)\rangle=\langle S \circ T, \nu\rangle=\int F_{S} d \nu
$$

Then

$$
\begin{aligned}
\left\langle S, T\left(\gamma_{\alpha} \mu\right)\right\rangle & =\int F_{S} \gamma_{\alpha} d \mu \\
& =\int \gamma_{\alpha} d\left(F_{S} \mu\right) \rightarrow \int f d\left(F_{S} \mu\right), \text { and } \\
\int f d\left(F_{S} \mu\right) & =\int F_{S} d(f \mu)=\langle S \circ T, f \mu\rangle \\
& =\langle S, T(f \mu)\rangle=\left\langle S,(f \mu)^{\wedge}\right\rangle .
\end{aligned}
$$

The limit in (5.3) follows from the assuption that $\gamma_{\alpha} \rightarrow f$ weak*, and the equalities in (5.2)-(5.5) are from the definitions and (5.1). It now follows that $L_{\gamma_{\alpha}} \hat{\mu}=$ $T\left(\gamma_{\alpha} \mu\right) \rightarrow T(f \mu)=(f \mu)^{\wedge} \in L^{\infty}(\Gamma)$ weakly. The equivalence of (1) and (2) is now an exercise in functional analysis. The last assertion is immediate from the equivalence of (1) and (2).

A similar argument yields the following generalization. Its proof is essentially identical to the proof of Theorem 5.1 (just remove the " $" \mathrm{~s}$ ), and is left to the reader.

Theorem 5.2. Let $G$ be an infinite locally compact abelian group. Let $\mu \in M(G)$, $\mu \neq 0$. Let $X$ be a Banach space, and let $T: L^{1}(\mu) \rightarrow X$ be a bounded linear mapping. Then $T(\{\gamma \mu: \gamma \in \Gamma\})$ is relatively weakly compact in $X$.

Furthermore, if $T$ is one-to-one, then the following are equivalent:

(1) $T(f \mu)$ is a weak accumulation point of $T(\{\gamma \mu: \gamma \in \Gamma\})$.

(2) $f$ is a weak ${ }^{*}$ accumulation point of $\{\gamma: \gamma \in \Gamma\}$ in $L^{\infty}(\mu)$.

$A$ non-abelian version of Theorem 5.1. Let $G$ be a locally compact group, not necessarily abelian. Let $C B(G)$ be the set of continuous bounded functions on $G$. Let $B(G)$ denote the subspace of $C B(G)$ that is generated by the continuous positive definite functions [18, Vol. II, p. $253 \mathrm{ff}$ ]. Each $f \in B(G)$ is thus a sum

$$
f=p_{1}-p_{2}+i\left(p_{3}-p_{4}\right), \quad p_{1}, \ldots, p_{4} \text { positive-definite. }
$$

The Eberlein proof [9], [5, p. 37] shows that every function in $B(G)$ is weakly almost periodic on $G$. 
Proposition 5.3. Let $\Gamma$ be a locally compact group and $f \in B(\Gamma)$. Then $B(\Gamma)$ is closed under the operation of taking weak limits in $C B(\Gamma)$ of translates of its elements.

Proof. Let $K_{p}$ denote the pointwise closure of convex hull of the left orbit of $f$. Then, $K_{p}$ must be contained in $B\left(\Gamma_{d}\right)$ (here $\Gamma_{d}$ is $\Gamma$ with the discrete topology. This is because the weak* topology on bounded sets of $B(\Gamma)$ is the same as the pointwise topology on $B\left(\Gamma_{d}\right)$. Now, the weak closure of the translates of $f$, call it $K$, is weakly compact. Also $K$ is contained in $C B(\Gamma)$. It follows that the weak topology agrees with the pointwise topology. So $K=K_{p}$. Consequently, $K$ is contained in the intersection of $B\left(\Gamma_{d}\right) \cap C B(\Gamma)$. By a result in Eymard [12, 2.24], $K$ must be contained in $B(\Gamma)$. In particular, any weak limit of translates of $f$ must belong to $B(\Gamma)$.

The following result led us to Theorem 5.2 .

Corollary 5.4. Let $\Gamma$ be a locally compact abelian group with dual group $G$. Let $M(G)$ be the space of bounded regular Borel measures on $G$. Let $\mu \in M(G)$. Then the set $O^{(\times)}(\mu)=\{\gamma \mu: \gamma \in \Gamma\}$ is relatively weakly compact in $M(G)$.

Proof of Corollary 5.4. Let $X=M(G)$ and $T$ the identity mapping. Then the conclusion is immediate from Theorem 5.2

$$
\text { 6. Orbits of } \gamma f \text { AND } \delta_{x} * f \text { FOR } f \in A_{p} \text { AND } f \in M_{p}
$$

This section gives some " $p$ " versions of Theorem 4.1 .

If $G$ is a locally compact abelian group, and $1 \leq p<\infty$, an $L^{p}$-multiplier is a linear mapping of $L^{p}(G)$ that commutes with translations. Every bounded regular Borel measure on $G$ gives rise to an $L^{p}$-multiplier by convolution. We let $M_{p}(G)$ denote the set of $L^{p}$-multipliers on $G$ with the operator norm. Then $M_{p}(G)$ is a Banach algebra. We begin with an immediate consequence of Theorem 5.2

Corollary 6.1. Let $\Gamma$ be a locally compact abelian group with dual group $G$. Let $\mu$ be a regular bounded Borel measure on $G$, considered as an operator on $L^{p}(G)$ by convolution. Then the set $O^{(\times)}(\mu)=\{\gamma \mu: \gamma \in \Gamma\}$ is relatively weakly compact in $M_{p}(G)$.

We now turn to some other versions of Theorem 4.1. The conclusion of the first is stronger than what we have in the general $L^{\infty}$ situation; contrast Remarks 4.2, above. The conclusion of the second is also stronger (in the opposite direction) than Theorem 4.1 there are some "new variant WAP" multipliers. We will restrict our discussion to the circle group $\mathbb{T}$ and its dual group $\mathbb{Z}$. We shall denote the $n^{\text {th }}$ character on $\mathbb{T}$ by $\chi_{n}(x)=e^{2 \pi i n x}, x \in \mathbb{T}, n \in \mathbb{Z}$.

We will assume that $1<p<\infty$. We begin by defining the predual of $M_{p}(\mathbb{T})$.

The Figa-Talamanca-Herz algebra 21] $A_{p}(G)$ is as follows: $f \in A_{p}(G)$ if and only if there exist $g_{j} \in L_{p}(G)$ and $h_{j} \in L^{q}(G)$ with

$$
\begin{aligned}
& f=\sum g_{j} * \check{h}_{j}, \text { where } \breve{h}(x)=h\left(x^{-1}\right) \text { and } \\
& \sum\left\|g_{j}\right\|_{p}\left\|h_{j}\right\|_{q}<\infty
\end{aligned}
$$

$p, q$ being conjugate indices. The norm is the infimum of all expressions (6.2) subject to (6.1). For all $G, A_{p}(G)$ is a regular Banach algebra [20. Of course, $A_{2}(G)=A(G)$, the usual Fourier algebra. The dual space of $A_{p}(\mathbb{T})$ can be identified 
with the space $M_{p}=M_{p}(\mathbb{T})$ of $L^{p}(\mathbb{T})$-multipliers; see [15, 10.2.16]. Each regular bounded Borel measure on $\mathbb{T}$ gives rise to a multiplier. In particular, we will be interested in the absolutely continuous measures, and in the discrete measures. First, though, we consider $A_{p}(\mathbb{T})$.

Proposition 6.2. Let $f \in A_{p}(\mathbb{T}), f \neq 0$. Let $\left\{\gamma_{k}\right\}$ be an infinite subset of $\mathbb{Z}$. Then $\left\{\gamma_{k} f: k \in \mathbb{Z}\right\}$ has no infinite subsequence $\left\{\gamma_{\ell} f: 1<\ell<\infty\right\}$ that is relatively weakly compact.

We need some Littlewood-Paley theory for the proof of Proposition 6.2, We let

$$
E_{j}= \begin{cases}\left\{n: 2^{j-1} \leq n<2^{j}\right\} & \text { if } j>0, \\ \{0\} & \text { if } j=0, \text { and } \\ \left\{n:-2^{j}<n \leq-2^{j-1}\right\} & \text { if } j<0 .\end{cases}
$$

We use $\left\{E_{j}\right\}_{j=-\infty}^{\infty}$ for the resulting Littlewood-Paley decomposition of $\mathbb{Z}$, and $S_{j} f=$ $\sum_{\lambda \in E_{j}} \hat{f}(\lambda) \lambda$. The book [11] has a proof that for $1<p<\infty$, there exist constants $0<A_{p}<B_{p}<\infty$ with

$$
A_{p}\|f\|_{p} \leq\left\|\left(\sum_{j=-\infty}^{\infty}\left|S_{j} f\right|^{2}\right)^{1 / 2}\right\|_{p} \leq B_{p}\|f\|_{p} \text { for all } f \in L_{p}(\mathbb{Z}) .
$$

The following lemma is nearly immediate from (6.4); see [15, 10.1-10.2] for details.

Lemma 6.3. Let $\left\{c_{j}\right\} \in \ell^{\infty}$. Then $\left\|\sum_{j} c_{j} S_{j}\right\|_{A_{p}(T)^{*}} \leq C_{p} \sup \left|c_{j}\right|$, where the constant $C_{p}$ depends only on $p$.

Proof of Proposition 6.2. Suppose first that $f(0)=1$. It is apparent from the definition of $A_{p}(\mathbb{T})$ that there exists a trigonometric polynomial $t$ such that $t(0)=$ 1 and $\|f-t\|_{A_{p}}<\frac{1}{9 C_{p}}$, where $C_{p}$ is given by Lemma 6.3. Let $T$ denote the support of the Fourier transform of $t$. Let $\left\{\gamma_{k(\ell)}\right\}$ be an infinite subsequence of $\left\{\gamma_{k}\right\}$. We will show that $\left\{\gamma_{k(\ell)} f\right\}$ has a subsequence $\left\{\gamma_{m(\ell)} f\right\}$ which is not relatively weakly compact.

We claim it will be enough to show that $\gamma_{\ell} f$ does not accumlulate weakly at 0 . Indeed, since $\left\{\gamma_{m(\ell)} f\right\}$ converges to zero as far as the absolutely continuous measures are concerned, and since evaluation at the absolutely continuous measures separates points of $A_{p}(\mathbb{T})$, it will be necessary for $\left\{\gamma_{m(\ell)} f\right\}$ to accumulate weakly at 0 , if $\left\{\gamma_{m(\ell)} f\right\}$ is to be relatively weakly compact. Thus it will be enough to construct an element $S$ of $M_{p}(\mathbb{T})=A_{p}(\mathbb{T})^{*}$ such that

$$
\liminf \left|\left\langle S, \gamma_{\ell} f\right\rangle\right|>0 \text {. }
$$

We may assume that $m(\ell)$ is increasing so rapidly that there exist $j(1)<j(2)<$ ... such that, for all $\ell \geq 1$,

$$
\begin{aligned}
& m(\ell) \in E_{j(\ell)} \text { and } \\
& m(\ell)+T \subset E_{j(\ell)-1} \cup E_{j(\ell)} \cup E_{j(\ell)+1} .
\end{aligned}
$$


Let $\left\{c_{\ell}\right\} \in \ell^{\infty}$. Then

$$
\begin{aligned}
& \sum_{\ell} c_{\ell}\left(S_{j(\ell)-1}+S_{j(\ell)}+S_{j(\ell+1)}\right) \in M_{p}(\mathbb{T}) \text { and } \\
& \left\|\sum_{\ell} c_{\ell}\left(S_{j(\ell)-1}+S_{j(\ell)}+S_{j(\ell+1)}\right)\right\|_{M_{p}} \leq C_{p} \sup _{\ell}\left|c_{\ell}\right|,
\end{aligned}
$$

where $C_{p}$ is given by Lemma 6.3. Also, for all $1 \leq k_{0}<\infty$,

$$
\begin{aligned}
& \left\langle\sum_{\ell} c_{\ell}\left(S_{j(\ell)-1}+S_{j(\ell)}+S_{j(\ell+1)}\right), \gamma_{k_{0}} t\right\rangle=c_{k_{0}} \text { and } \\
& \left|\left\langle\sum_{\ell} c_{\ell} S_{j(k)}, \gamma_{k_{0}}(t-f)\right\rangle\right|<\frac{\sup \left|c_{k}\right|}{9} .
\end{aligned}
$$

Taking $c_{\ell}=1$ for all $\ell$, and $S=\sum_{\ell} c_{\ell}\left(S_{j(\ell)-1}+S_{j(\ell)}+S_{j(\ell+1)}\right)$, we see that (6.5) holds, and $\left\{\gamma_{k} f\right\}$ is not relatively weakly compact.

Now suppose that $f \neq 0$, but $f(0)=0$. Then there is an $x$ such that $f^{\prime}=\delta_{x} * f$ has $f^{\prime}(0) \neq 0$. We may assume $f^{\prime}(0)=1$, so there exists, by the first part of the proof, $\gamma_{k}$ such that $\left\{\gamma_{k} f^{\prime}\right\}$ has no infinite relatively weakly compact subsets. Then $\delta_{-x} *\left(\gamma_{k} \delta_{x} * f\right)=\left\langle x, \gamma_{k}\right\rangle f$ for all $k$. We may replace $\gamma_{k}$ with a subsequence $\gamma_{k(\ell)}$ such that $\left\langle x, \gamma_{k(\ell)}\right\rangle$ converges. Suppose that $\left\{\gamma_{k(\ell)} f\right\}$ has a relatively weakly compact subset, which we may as well assume to be $\left\{\gamma_{k(\ell)} f\right\}$. Then

$$
\left\{\left\langle x, \gamma_{k(\ell)}\right\rangle\right\} \times\left\{\gamma_{k(\ell)} f: k \geq 1\right\}
$$

is relatively compact in $\mathbb{T} \times L^{\infty}(\mu)^{* *}$, when the second factor has the weak topology, and so the diagonal $\left\{\left(\left\langle x, \gamma_{k(\ell)}\right\rangle, \gamma_{k(\ell)} f\right)\right\}$ is also relatively compact in $\mathbb{T} \times L^{\infty}(\mu)^{* *}$. Therefore, the continuuous image of that diagonal, $\left\{\left\langle x, \gamma_{k(\ell)}\right\rangle \gamma_{k(\ell)} f: k \geq 1\right\}$, is relatively weakly compact, a contradiction. Thus, $\left\{\gamma_{k(\ell)} f\right\}$ has no relatively weakly compact subsets.

A similar argument yields the analogous result for all groups for which there is a Littlewood-Paley theorem (e.g, $\mathbb{R})$.

We now turn to two versions of Theorem 4.1 for the space $M_{p}=M_{p}(\mathbb{T})$ of $L^{p}(\mathbb{T})$ multipliers. Each multiplier $M$ has a Fourier transform, denoted $\widehat{M}$. We let $H$ be the element of $M_{p}(\mathbb{T})$ whose Fourier transform is the characteristic function of the set of non-negative integers. We first look at the translation of the transforms. In this circumstance, we find some multipliers that are "WAP" and some that are not.

Proposition 6.4. (1) Let $M \in M_{p}(\mathbb{T})$ be in the closure of the multipliers whose transforms have finite support. Let $\left\{\gamma_{k}\right\}$ be any infinite subset of $\mathbb{Z}$. Then $\{\gamma M: \gamma \in \mathbb{Z}\}$ converges weakly to 0 .

(2) The only relatively weakly compact subsets of $\{\gamma H: \gamma \in \mathbb{N}\}$ are the finite sets.

(3) If $\mu \in M(\mathbb{T})$, then $\{\gamma \mu: \gamma \in \Gamma\}$ is weakly compact in $M_{p}(\mathbb{T})$.

Proof. (1) We need a few facts about $M_{p}^{*}$. Consider any sequence $\left\{\gamma_{\ell}\right\}$ such that

$$
1 \leq \gamma_{1} \text { and } 2 \gamma_{\ell} \leq \gamma_{\ell+1} \text {. }
$$

Because of (6.12), we may apply [15, 10.3.7] to conclude that there exists $T_{o} \in M_{p}$ such that

$$
\widehat{T}_{o}(n)= \begin{cases}1, & \text { for } n \in\left\{\gamma_{\ell}: \ell \geq 1\right\} \\ 0, & \text { otherwise }\end{cases}
$$


Using Lemma 6.3 and (6.13), we see that for each sequence $\left\{c_{\ell}\right\}$ of complex numbers, $\sum_{\ell} c_{\ell} \gamma_{\ell} \in A_{p}(\mathbb{T})^{*}=M_{p}(\mathbb{T})$ and $\left\|\sum_{\ell} c_{\ell} \gamma_{\ell}\right\|_{M_{p}} \leq C_{p} \sup \left|c_{\ell}\right|$. Therefore the set of "restrictions" of the elements of the dual of the multipliers to $\left\{\gamma_{\ell}\right\}$ is isomorphic to $\ell^{1}$, that is, if $X \in M_{p}^{*}$, then

$$
\sum_{\ell}\left|\widehat{X}\left(\gamma_{\ell}\right)\right|<\infty \text {, so } \widehat{X}(n) \rightarrow 0 \text { as }|n| \rightarrow \infty .
$$

Now suppose that $M$ is a $L^{p}(\mathbb{T})$-multiplier whose Fourier transform has finite support, say Support $M \subset[-k, k]$. Let $X \in M_{p}^{*}$. Then

$$
\left|\left\langle X, \gamma_{\ell} M\right\rangle\right| \leq(2 k+1) \sup _{j}|\widehat{M}(j)| \sup _{|n| \geq \ell}|\widehat{X}(n)| \rightarrow 0 \text { as } \ell \rightarrow \infty,
$$

by (6.14). Hence, $\gamma_{\ell} M \rightarrow 0$ weakly.

If $M$ is in the closure of the multipliers whose transforms have finite support, then a $2 \epsilon$ argument shows that $\left|\left\langle X, \gamma_{\ell} M\right\rangle\right| \rightarrow 0$ as $\ell \rightarrow \infty$, as required.

(2) The argument here is basically that of Theorem 4.1; we look at an element of the maximal ideal space of the algebra. We first note that for each $f \in A_{p}(\mathbb{T})$,

$$
\lim _{n \rightarrow \infty}\left\langle\chi_{n} H, f\right\rangle=0 \text { and } \lim _{n \rightarrow-\infty}\left\langle\chi_{n} H, f\right\rangle=\left\langle\delta_{0}, f\right\rangle=f(0) .
$$

Thus,

$$
\chi_{n} H \rightarrow 0 \text { as } n \rightarrow \infty \text { and } \chi_{n} H \rightarrow \delta_{0} \text { as } n \rightarrow-\infty,
$$

in the weak* topology $\sigma\left(M_{p}, A_{p}\right)$. In an abuse of notation, let $\chi_{n}$ also denote the element $f \in A_{p}(\mathbb{T})$ with $f(x)=\chi_{n}(x), x \in \mathbb{T}, n \in \mathbb{Z}$. Let $\Xi_{+}$be any weak* accumulation point of $\left\{\chi_{n}: n \geq 0\right\}$ in $M_{p}^{*}$ (this is in the $\sigma\left(M_{p}^{*}, M_{p}\right)$ topology!), and let $\Xi_{-}$be any weak* accumulation point of $\left\{\chi_{n}: n \leq 0\right\}$. Then

$$
\left\langle\Xi_{+}, \chi_{n} H\right\rangle=1 \text { for } n \in \mathbb{Z} \text {, and }\left\langle\Xi_{-}, \chi_{n} H\right\rangle=0 \text { for } n \in \mathbb{Z} .
$$

We separate the positive and negative directions. Positive: let $L_{+}$be any weak accumulation point of $\left\{\chi_{n} H: n \geq 0\right\}$. Then the first equalities of (6.15) and (6.16) are contradictory: $\chi_{n} H$ cannot have a weak accumulation point in $M_{p}$ as $n \rightarrow+\infty$. (Indeed, $\left\langle\Xi_{+}, L_{+}\right\rangle=1$, so $\left\|L_{+}\right\|_{A_{p}} \geq 1$. On the other hand, $\chi_{n} H \rightarrow 0$ weak* in $M_{p}$ as $n \rightarrow \infty$, and weak convergence implies weak* convergence. Hence $L=0$, a contradiction.)

Negative: let $L_{-}$be any weak accumulation point of $\left\{\chi_{n} H: n \leq 0\right\}$. Then the second equalities of (6.15) and (6.16) are contradictory.

(3) This is Corollary 6.1

If we use translation in $\mathbb{T}$, we have a different result.

Proposition 6.5. Let $M=\delta_{0} \in M_{p}(\mathbb{T})$ be the unit point mass at the identity. Then $\left\{\delta_{x} * M: x \in \mathbb{T}\right\}$ is not relatively weakly compact in $M_{p}$.

Proof. Let $\{x(j)\}$ be a sequence that converges to 0 . We shall show that $\left\{\delta_{x(j)}\right\}$ is not relatively weakly compact in $M_{p}(\mathbb{T})$. By evaluating the translates $\delta_{x}$ at elements of $A_{p}(\mathbb{T})$, we see that if $\left\{\delta_{x(j)}\right\}$ is weakly compact, then we must have $\delta_{x(j)} \rightarrow \delta_{0}$ weakly. We will show that this does not occur. Indeed, by passing to a subsequence, we may assume that each finite subset of $U=\left\{x_{j}\right\}$ is a Helson set with Helson constants bounded by 2 (see, e.g., [15, p. 34ff] for this and the definition of "Helson"). Then for every finite subset $V \subset U$ and complex numbers $c_{v}, v \in V$, there exists $f \in A(\mathbb{T})=A_{2}(\mathbb{T})$ such that $f(v)=\bar{c}_{v}$ for all $v \in V$ and 
$\|f\|_{A(\mathbb{T})} \leq 2 \sup _{v \in V}\left|c_{v}\right|$ (this follows from, e.g., [32, 5.1.3]). Since $A_{2}(\mathbb{T}) \hookrightarrow A_{p}(\mathbb{T})$ is norm-decreasing, we can take weak* limits to conclude that for every bounded set $\left\{c_{u} \in \mathbb{C}: u \in U\right\}$, there exists $F \in M_{p}(\mathbb{T})^{*}$ such that $\left\langle F, \delta_{u}\right\rangle=c_{u}, u \in U$ with $\|F\|_{M_{p}^{*}} \leq 2 \sup _{u}\left|c_{u}\right|$. It follows that $\left\{\delta_{u}: u \in U\right\}$ does not converge to $\delta_{0}$ weakly, and, therefore, that $\left\{\delta_{u}: u \in U\right\}$ is not relatively weakly compact.

Corollary 6.6. $\left\{\delta_{x} * H: x \in \mathbb{T}\right\}$ is not relatively weakly compact in $M_{p}$.

Proof. Indeed, suppose that $\left\{\delta_{x} * H: x \in \mathbb{T}\right\}$ is relatively weakly compact. Let $H^{\prime}$ be the element of $M_{p}$ whose Fourier transform is the characteristic function of $(-\infty,-1)$, so $H+H^{\prime}=\delta_{0}$. Then $\left\{\delta_{y} * H^{\prime}: y \in \mathbb{T}\right\}$ would also be relatively weakly compact, as would be the product $\left\{\delta_{x} * H: x \in \mathbb{T}\right\} \times\left\{\delta_{y} * H^{\prime}: y \in \mathbb{T}\right\}$ (in $\left.M_{p} \times M_{p}\right)$ and the diagonal $\left\{\left(\delta_{x} * H, \delta_{x} * H^{\prime}\right): x \in \mathbb{T}\right\}$. Since addition is continuous, $\left\{\delta_{x} * H+\delta_{x} * H^{\prime}: x \in \mathbb{T}\right\}=\left\{\delta_{x}: x \in \mathbb{T}\right\}$ would be relatively weakly compact, contradicting Proposition 6.5.

\section{ORBITS OF $\delta_{x} * f$ AND $\gamma f$ FOR $f \in L^{1}(G)$}

In this section, we consider $L^{1}(G)$ as our source of functions to be translated or multiplied. We begin with a simple observation.

Remark 7.1. Let $G$ be a locally compact group with dual group $\Gamma$ and $f \in L^{1}(G)$, $f \neq 0$. Let $O^{(*)}(f)=\left\{\delta_{x} * f: x \in G\right\}$ be the set of translates of $f$. Then the following are equivalent:

(1) $O^{(*)}(f)$ is relatively weakly compact in $L^{1}(G)$ (i.e., relatively compact in the $\sigma\left(L^{1}(G), L^{\infty}(G)\right)$ topology).

(2) $G$ is compact.

(3) $O^{(*)}(f)$ is norm compact.

Furthermore, if $G$ is non-compact, then the only relatively weakly compact subsets of $O^{(*)}(f)$ are those sets $O_{E}^{(*)}(f)=\left\{\delta_{x} * f: x \in E\right\}$ with $E$ relatively compact in $G$ (which are also relatively norm compact).

Proof. (1) $\Rightarrow(2)$. We suppose that $G$ is not compact. Then whatever the net $x_{\alpha} \rightarrow \infty, \delta_{x_{\alpha}} * f \rightarrow 0$ weak $^{*}$, i.e., in the $\sigma\left(L^{1}(G), C_{o}(G)\right)$ topology. Since $f \neq 0$, there exists $S \in L^{\infty}(G)$ and $\alpha_{n} \rightarrow \infty$ such that $\int_{G} \delta_{x_{\alpha_{j}}} f(y) S(y) d y \rightarrow\|f\|_{1}$, so $\delta_{x_{\alpha_{j}}} * f \nrightarrow \rightarrow 0$. Hence, if $G$ is not compact, then $O^{(*)}(f)$ is not relatively weakly compact, since the weak and weak* topologies do not agree on $O^{(*)}(f)$.

$(2) \Rightarrow(3)$ is trivial, since translation in $L^{1}(G)$ is norm continuous.

$(3) \Rightarrow(1)$ is trivial.

The final statement is immediate from the proof of $(1) \Rightarrow(2)$, except for part of the parenthetical remark, which is immediate from $(2) \Rightarrow(3)$.

Proposition 7.2. Let $G$ be a locally compact abelian group with dual group $\Gamma$. Let $f \in L^{1}(G), f \neq 0$. Let $O^{(\times)}(f)=\{\gamma f: \gamma \in \Gamma\}$. Then the following hold:

(1) $G$ is discrete $\Leftrightarrow O^{(\times)}(f)$ is norm compact.

(2) $O^{(\times)}(f)$ is relatively weakly compact (in the $\sigma\left(L^{1}(G), L^{\infty}(G)\right.$ ) topology).

(3) If $G$ is not discrete, then $O^{(\times)}(f) \cup\{0\}$ is weakly compact, but not norm compact. 
Proof. (1) Taking Fourier transforms, we see that we are considering translation in the Fourier algebra $A(\Gamma)$. If $G$ is compact, then the continuity of translation implies that $O^{(\times)}(f)$ is norm compact.

Suppose that $O^{(\times)}(f)$ is relatively norm compact. Then $O^{(\times)}(f)$ would also be relatively weakly compact and the two topologies would agree on $O^{(\times)}(f)$. Suppose also that $G$ is not discrete, so $\Gamma$ is not compact. Then if $\gamma_{\alpha}$ is a net in $\Gamma$ with $\gamma_{\alpha} \rightarrow \infty$, then $\gamma_{\alpha} f \rightarrow 0$ weakly, by the Riemann-Lebesgue Lemma. Hence, if $O^{(\times)}(f)$ is norm compact, then $\gamma_{\alpha} f$ only would accumulate at 0 . But we can find $\epsilon>0$ and a compact subset $E \subset G$ such that $|f|>\epsilon$ on $E$, and the Haar measure $m_{G}(E)>\epsilon$. Suppose that $\gamma_{\alpha} f$ accumulated (in norm) at $\gamma$. Then $\gamma_{\alpha} \chi_{E}$ would accumulate (in norm) at $\gamma \chi_{E} / f_{\mid E}$. Also, the norm convergence implies that $\gamma_{\alpha} \chi_{E}$ would have absolute value 1 a.e. on $E$. Hence $|\gamma| \geq \epsilon$ on $E$, so the norm and weak topologies do not agree on $O^{(\times)}(f)$. That completes the proof of (1).

(2) This is a special case of Corollary 5.4

(3) The failure of norm compactness is immediate from (1).

We must identify the weak closure of $O^{(\times)}(f)$, when $G$ is not discrete. Let $S \in L^{\infty}(\Gamma)=L^{1}(G)^{*}$. Then

$$
\langle S, \gamma f\rangle=\int_{G}\langle\gamma, x\rangle f(x) S(x) d x \rightarrow 0 \text { as } \gamma \rightarrow \infty,
$$

by the Riemann-Lebesgue Lemma, applied to the $L^{1}$-function $f S$. It follows that the weak closure of $O^{(\times)}(f)$ is indeed $O^{(\times)}(f) \cup\{0\}$.

The reader will note that the last paragraph of the preceding proof is a simple version of Lemma 2.2. as well as being closely related to the argument of Theorem 5.1 .

\section{Orbits of $\gamma f$ AND $\delta_{x} * f$, FOR $f \in C_{o}(G)$}

Multiplication in $C_{o}(G)$ characterizes discreteness, as follows.

Proposition 8.1. Let $G$ be a locally compact abelian group. Let $f \in C_{o}(G), f \neq 0$. Then the following are equivalent:

(1) $G$ is discrete.

(2) $O^{(\times)}(f)=\{\gamma f: \gamma \in \Gamma\}$ is relatively norm compact in $C_{o}(G)$.

(3) $O^{(\times)}(f)=\{\gamma f: \gamma \in \Gamma\}$ is relatively weakly compact in $C_{o}(G)$.

Proof. (1) $\Rightarrow(2)$ is trivial: if $\gamma_{\alpha} \in \Gamma$ is any net, then it has a subnet $\gamma_{\alpha(n)}$ that converges pointwise on the (countable) support of $f$. Hence, $\gamma_{\alpha(n)} f$ converges pointwise and boundedly on $G$. A two epsilon argument, using $f \in C_{o}(G)$, shows that $\gamma_{\alpha(n)} f$ converges in norm. A fortiori, the net converges weakly, i.e., in $\sigma\left(c_{o}(G), \ell^{1}(G)\right)$.

$(2) \Rightarrow(3)$ is trivial.

$(3) \Rightarrow(1)$. Suppose $G$ is not discrete. Let $\mu$ be an absolutely continuous measure whose support is the support of $f$. Suppose that $\gamma_{\alpha}$ is a net in $\Gamma$ that tends to infinity in $\Gamma$. Then $\gamma_{\alpha} f \rightarrow 0$ weak* in $L^{\infty}(\mu)$ (by the Riemann-Lebesgue Lemma). Of course, this holds for any $\nu$ that is absolutely continuous with respect to $\mu$. Hence, if $\gamma_{\alpha} f$ converges weakly, it converges weakly to 0 .

On the other hand, let $\omega$ be a Dirichlet probablity measure whose support is contained in the support of $f$. Then there exists a net $\gamma_{\alpha} \rightarrow \infty$ such that $\omega\left(\gamma_{\alpha}\right) \rightarrow$ $\|\omega\|=1$. Hence $\gamma_{\alpha} f \rightarrow f$ weak $^{*}$ in $L^{\infty}(\omega)$, so if $\gamma_{\alpha} f$ has a weak accumulation 
point, 0 is not that weak accumulation point. Hence, $\gamma_{\alpha} f$ does not have a weak accumulation point.

Remark 8.2. For completeness, we make the trivial observations: let $G$ be a locally compact abelian group and $f \in C_{o}(G), f \neq 0$. Then

(1) $\left\{\delta_{x} * f: x \in G\right\}$ is norm compact iff $G$ is compact.

(2) 0 is in the weak closure of $\left\{\delta_{x} * f: x \in G\right\}$ iff $G$ is not compact. In this case, $\left\{\delta_{x} * f: x \in G\right\}$ is relatively weakly compact, with 0 as the "point at infinity".

\section{WEAK* LIMITS AND THE TOPOLOGY OF $\Gamma(\mu)$}

We assume in this section that $G$ is a locally compact abelian group with dual group $\Gamma$, and that $\mu$ is a finite regular measure on $G$.

For each $\mu \in M(G)$, the space of bounded regular Borel measures, let $\Gamma(\mu)$ denote the image of $\Gamma$ in $L^{\infty}(G)$, let $\overline{\Gamma(\mu)}$ be the closure of $\Gamma(\mu)$ in $L^{\infty}(|\mu|)$ in the weak* topology, and let $\tilde{\Gamma}(\mu)$ be the set of elements $\chi \in \overline{\Gamma(\mu)}$ with $|\chi|=1$ a.e. $\mathrm{d} \mu$. See [24, pp. 13, 33, 37].

It follows that $\overline{\Gamma(\mu)}$ is a compact semi-topological semigroup (in the weak* topology): multiplication (pointwise of functions) is separately continuous. Thus, $\overline{\Gamma(\mu)}$ is a semigroup compactification of $\Gamma$ (see 2] ). It is natural to ask the following question: given a measure $\mu$, what can we say about $\overline{\Gamma(\mu)}$ ? This question has been considered quite extensively in 24, leading (among other things) to a new proof of the Cohen Idempotent Theorem and its generalization to non-abelian groups (see [23]).

The main results of this section are as follows. First, the simple result that $\Gamma(\mu)$ and $\tilde{\Gamma}(\mu)$ are topological groups (multiplication is continuous in both variables simultaneously), but $\overline{\Gamma(\mu)}$ is (in general) only a semitopological semigroup; see Theorem 9.4.

We show that measures with finite support group are not interesting in this context in Proposition 9.2 .

We consider when $\Gamma(\mu)$ can be metrizable in Proposition 9.3

Discrete measures are characterized in terms of properties of $\overline{\Gamma(\mu)}$ (Theorem 9.4).

We answer the question of when $\tilde{\Gamma}(\mu)$ or $\Gamma(\mu)$ can be discrete in Theorem 9.5

We will use the notion of "L-subalgebra": a norm closed subspace of measures which is closed under the operation of multiplication by bounded measurable functions.

Proposition 9.1. Let $G$ be a locally compact abelian group and $\mu \in M(G)$. Then the multiplications in $\Gamma(\mu)$ and $\tilde{\Gamma}(\mu)$ are continuous in both variables simultaneously.

Proof. This is a special case of Corollary 2.8. It is also a special case of Proposition 11.5

Proposition 9.2. Let $\mu$ be a regular probability measure on the locally compact abelian group $G$ with dual group $\Gamma$. Let $A$ be the symmetric L-subalgebra of $M(G)$ generated by $\mu$, and let $C(\overline{\Gamma(\mu)})$ be the space of bounded continous functions on $\overline{\Gamma(\mu)}$. Then the following are equivalent:

(1) The support of $\mu$ generates an infinite group. 
(2) $\Gamma(\mu)$ is infinite.

(3) $C(\overline{\Gamma(\mu)})$ is infinite dimensional.

(4) $C(\overline{\Gamma(\mu)}) / L^{1}(\mu)^{-}$has dimension at least $c$.

(5) $\widehat{A}$ is not closed in $C(\Gamma(\mu))$.

Proof. The equivalence of (1)-(3) is trivial: $\Gamma(\mu)$ is finite if and only if $L^{1}(\mu)$ and $L^{1}(\mu)^{\varsigma}$ are finite dimensional, so $C(\overline{\Gamma(\mu)})$ is finite dimensional and $C(\overline{\Gamma(\mu)})=$ $L^{1}(\mu)$. [Because $\Gamma(\mu)$ separates the points of $L^{1}(\mu)$, that latter Banach space is finite dimensional if and only if $\Gamma(\mu)$ is finite if and only if the support of $\mu$ generates an infinite group.]

$(2) \Rightarrow(4)$. We prove more, namely that $(2)$ implies that $C(\overline{\Gamma(\mu)}) / \widehat{A}$ has dimension at least $c$, where $A$ is the symmetric L-subalgebra generated by $\mu$.

Of course $\widehat{A}$ is dense in $C(\overline{\Gamma(\mu)})$ if $A$ contains an identity, and otherwise the closure of $\widehat{A}$ has codimension one in $C(\overline{\Gamma(\mu)})$, by the Stone-Weierstrass Theorem.

If $\widehat{A}$ were not closed in $C(\overline{\Gamma(\mu)})$, then $C(\overline{\Gamma(\mu)}) / \widehat{A}$ has dimension at least $c$ by a standard theorem in Banach space theory.

If $\widehat{A}$ is closed in $C(\overline{\Gamma(\mu)})$, then the uniform topology on $\widehat{A}$ and the $L^{1}$ norm topology are equivalent, so $\widehat{A}$ is weakly sequentially complete. Since $\widehat{A}$ is a $\mathrm{C}^{*}$ subalgebra of $C(\overline{\Gamma(\mu)})$, it must be finite dimensional [7]. Thus, $\Gamma$ is finite, so $G$ is finite.

We note that we have also proved that $(2) \Longrightarrow(5)$.

That (4) and (5) each imply (2) is trivial.

Proposition 9.3. The following hold for $\mu \in M(G)$, where $G$ is a locally compact abelian group:

(1) $\Gamma(\mu)$ is metrizable if $L^{1}(\mu)$ is separable; the latter occurs if $G$ is separable.

(2) $\Delta(M(G))$ is metrizable iff and only if $G$ is countable.

Proof. (1) This is trivial. (But $\Gamma(\mu)$ can be metrizable when $G$ is not separable: let $G$ be compact and not separable and let $\mu$ be a Haar measure on $G$.)

(2) If $G$ is not countable, then we can map $\Delta(M(G))$ continuously onto the Bohr compactification $b \Gamma$ of $\Gamma$ by the mapping restricting each multiplicative linear functional on $M(G)$ to the discrete measures. If $G$ is not countable, then $b \Gamma$ is not metrizable, so neither is $\Delta(M(G))$.

Theorem 9.4. Let $G$ be a locally compact abelian group with dual group $\Gamma$ and $\mu \in M(G), \mu \geq 0$. Then the following are equivalent:

(1) $\mu$ is discrete.

(2) The multiplication in $\overline{\Gamma(\mu)}$ is jointly continuous.

(3) $\tilde{\Gamma}(\mu)$ is compact.

Furthermore, if $\Gamma(\mu)$ is compact, then $\mu$ is discrete (though the converse is false).

Proof. (1) $\Leftrightarrow(2)$. If $\mu$ is discrete, then $\overline{\Gamma(\mu)}=\tilde{\Gamma}(\mu)$ is just a quotient of the Bohr compactification of $\Gamma$, so the multiplication is jointly continuous.

But, if $\mu$ is not discrete, then Lemma 2.2 applies, so there exists a net $\gamma_{\alpha}$ which converges weak* to an element of $\overline{\Gamma(\mu)}$ which is zero with respect to the continuous part of $\mu$. This implies that the limit of the product is not the product of the limits: $\lim _{\alpha} \gamma_{\alpha} \bar{\gamma}_{\alpha}=1$ everwhere, but $\lim _{\beta} \lim _{\alpha} \gamma_{\alpha} \bar{\gamma}_{\beta}=0$ (with respect to the continuous part of $\mu$ ). Therefore the multiplication is not jointly continuous. 
$(1) \Leftrightarrow(3)$. If $\mu$ is discrete, then, as in the proof of $(1), \Gamma(\mu)$ is compact, so $\overline{\Gamma(\mu)}=\Gamma(\mu)=\tilde{\Gamma}(\mu)$ is compact. On the other hand, suppose that $\tilde{\Gamma}(\mu)$ is compact. Let $H=(\tilde{\Gamma}(\mu))$. If $\Lambda=(\tilde{\Gamma}(\mu))$ is compact, then $H$ is discrete. Now, $\hat{\mu}$ is a positive-definite, continuous function on $\Lambda$, so there exists a regular probability measure $\mu^{\prime}$ on $H$ with $\hat{\mu}^{\prime}=\hat{\mu}$ on $\tilde{\Gamma}(\mu)$. Since $\mu$ has the same Fourier-Stieltjes transform as $\mu^{\prime}, \mu=\mu^{\prime}$, that is, $\mu$ is supported on the discrete group $H$. The continuous group homomorphism of $\Gamma \rightarrow \tilde{\Gamma}(\mu)$ induces an injection of $H$ into $G$. Since $\mu$ is concentrated on (the image of) $H, \mu$ must be a discrete measure.

Finally, if $\Gamma(\mu)$ is compact, then $\Gamma(\mu)=\overline{\Gamma(\mu)}$ and $\Gamma(\mu)$ has jointly continuous multiplication. Apply the first part.

Now for a counterexample to show that the converse is false. We let $G=\mathbb{T}$ and $\mu=\delta_{\pi / \sqrt{2}}$. This gives us a discrete measure whose support group is (isomorphic to) $\mathbb{Z}$. Then $\Gamma(\mu)=\mathbb{Z}$ (not compact) and $\overline{\Gamma(\mu)}=\mathbb{T}$ (topologically).

Theorem 9.5. Let $G$ be a locally compact abelian group with dual group $\Gamma$ and $\mu \in M(G), \mu \geq 0$. Then the following hold:

(1) It is not the case that $G$ is necessarily discrete if $\Gamma(\mu)$ is compact.

(2) If $\tilde{\Gamma}(\mu)$ is countable, then $\Gamma(\mu)$ is discrete.

(3) If $\Gamma(\mu)$ is discrete, then $\Gamma(\mu)=\tilde{\Gamma}(\mu)$.

(4) There exist $\mu \in M(\mathbb{T})$ with $\Gamma(\mu)$ discrete and $\Gamma(\mu) \neq \overline{\Gamma(\mu)}$.

Proof. (1) Let $G$ be the Bohr compactification of $\mathbb{Z}$, and let $\mu$ be the unit point mass at the number $1 \in \mathbb{Z}$ (not the identity of $G$ ). Then $G$ is compact, and $\Gamma(\mu)=\mathbb{T}$ is also compact.

(2) If $\tilde{\Gamma}(\mu)$ is non-discrete, then it is a perfect space (because it is a topological group, so every point is non-isolated). But $\tilde{\Gamma}(\mu)$ is a subset of $L^{1}(\mu)$, and its relative (the norm!) topology agrees with the weak* topology by Lemma 2.6 Of course, if $\chi_{j}$ is Cauchy in $\tilde{\Gamma}(\mu)$ for the $L^{1}$-norm topology, then $\lim \chi_{j}$ must also be unimodular (that is, the absolute value is 1 everywhere), so $\tilde{\Gamma}(\mu)$ is closed in the $L^{1}$-norm topology. Hence $\tilde{\Gamma}(\mu)$ is a perfect complete metric space and therefore not countable [22, 6.65].

(3) This is an exercise in weak* limits. If $\Gamma(\mu)$ is discrete, then there exist $1 \leq$ $n<\infty$ and $\nu_{1}, \ldots, \nu_{n} \in L^{1}(\mu)$ such that $\{0\}=\left\{\gamma \in \Gamma(\mu): \sup _{j}\left|\hat{\nu}_{j}(\gamma)-\hat{\nu}_{j}(0)\right|<1\right\}$. Suppose $\gamma_{\alpha} \rightarrow \lambda$ is a net in $\Gamma(\mu)$ converging weak* to $\lambda \in \tilde{\Gamma}(\mu)$, with $\lambda \notin \Gamma(\mu)$. We can assume that for each $\beta$, the set $A_{\beta}$ of $\alpha$ with $\gamma_{\alpha} \neq \gamma_{\beta}$ is cofinal. [Indeed, just take a weak* neighbourhood $U$ of $\lambda$ that does not contain $\gamma_{\beta}$ and consider the set $A_{\beta}=\left\{\alpha: \gamma_{\alpha} \in U\right\}$. Then $A_{\beta}$ is cofinal.]

Then, for each $\beta$ and each $\alpha \in A_{\beta}, \sup _{j}\left|\hat{\nu}_{j}\left(\gamma_{\alpha} \bar{\gamma}_{\beta}\right)-\hat{\nu}_{j}(0)\right| \geq 1$. Taking first the weak* limit in $\alpha \in A_{\beta}$, and then using the continuity of the sup function (over a finite set), we have $\sup _{j}\left|\hat{\nu}_{j}\left(\lambda \bar{\gamma}_{\beta}\right)-\hat{\nu}_{j}(0)\right| \geq 1$. Now take the limit in $\beta$ : $\sup _{j}\left|\hat{\nu}_{j}(\lambda \bar{\lambda})-\hat{\nu}_{j}(0)\right| \geq 1$, which is absurd. Therefore, we cannot have $\Gamma(\mu) \neq \tilde{\Gamma}(\mu)$ when $\Gamma(\mu)$ is discrete.

(4) Consider the Riesz product $\mu=\lim _{n} \Pi_{k=1}^{n}\left(1+\cos 4^{k} x\right) d x$ on $\mathbb{T}$. (The limit is weak* .) Then $\overline{\Gamma(\mu)}$ can be identified topologically with $Z \times\left\{0, \frac{1}{2}, \frac{1}{4}, \frac{1}{8}, \ldots\right\}$, by Brown [3]; or see [15, 7.1.2].

Theorem 9.6. If $\Gamma(\mu)$ is open in $\tilde{\Gamma}(\mu)$ or open in $\overline{\Gamma(\mu)}$, then $\Gamma(\mu)=\tilde{\Gamma}(\mu)$. 
Proof. Suppose first that $\Gamma(\mu)$ is open in $\tilde{\Gamma}(\mu)$. Since the multiplication in $\tilde{\Gamma}(\mu)$ is separately continuous, all the cosets of $\Gamma(\mu)$ in $\tilde{\Gamma}(\mu)$ are also open. Hence, $\Gamma(\mu)$ is closed in $\tilde{\Gamma}(\mu)$. But $\Gamma(\mu)$ is dense in $\tilde{\Gamma}(\mu)$, so $\Gamma(\mu)=\tilde{\Gamma}(\mu)$.

If $\Gamma(\mu)$ is open in $\overline{\Gamma(\mu)}$, then $\Gamma(\mu)$ is open in $\tilde{\Gamma}(\mu)$ and hence equal to $\tilde{\Gamma}(\mu)$ by the preceding paragraph.

\section{Full measures And Dirichlet measures}

Let $G, H$ be locally compact abelian groups. Suppose that $H \stackrel{i}{\hookrightarrow} G$ continuously embeds $H$ as a subgroup of $G$. Let $\tau$ be the topology on $G$ which declares $i H$ to be an open subgroup of $G$, with the topology on $i H$ being that inherited from $H$. Let $G_{H}$ denote $G$ with that topology.

A non-negative regular Borel measure $\mu$ on the locally compact abelian group $G$ is full [24, p. 35] iff $\lim \sup _{\gamma \rightarrow \infty}|\hat{\mu}(\gamma)|<\|\mu\|$. A regular Borel measure $\mu$ on $G$ is full iff $|\mu|$ is full. A measure that is not full is called Dirichlet. These measures have already been mentioned in Remarks 4.2, Discrete measures and measures supported on Kronecker sets are Dirichlet.

Remark 10.1. If $H$ is the closed subgroup generated by the support of $\mu$, then $\Gamma(\mu)$ is topologically isomorphic to $\Gamma / H^{\perp}$ iff $\mu$ is a full measure in $M(H)$. This is 24, p. 35, Prop. 2].

Theorem 10.2. Let $G$ be a locally compact abelian group with dual group $\Gamma$ and $\mu \in M(G)$. If either $\Gamma(\mu)$ or $\tilde{\Gamma}(\mu)$ is locally compact, then there exists a locally compact group $H$ and a continuous embedding $H \stackrel{i}{\hookrightarrow} G$ of $H$ as a subgroup of $G$ such that

(1) $\mu \in M\left(G_{i H}\right)$,

(2) $\mu$ (as a measure on $G_{i H}$ ) is supported on $i H$,

(3) the support of $\mu$ (as a measure on $i H$ ) generates a dense subgroup of $i H$, and

(4) $\mu$ is a full measure on $i H$.

Proof. Suppose first that $\Gamma(\mu)$ is locally compact. Let $H$ be the dual group of $\Gamma(\mu)$. Then the natural mapping $r: \Gamma \rightarrow \Gamma(\mu)$ is onto, and $r$ induces a continuous embedding $H \stackrel{i}{\hookrightarrow} G$, which is one-to-one into. Furthermore $r$ is multiplicative.

First note that $\hat{\mu}$ as a function on $\Gamma(\mu)$ satisfies the Eberlein criterion [32, 1.9.1] to be the Fourier-Stieltjes transform of a measure $\nu$ on $H$. Furthermore, since $L^{1}(\mu)^{\wedge}$ separates points of $\Gamma(\mu)$, the group generated by the support of $\nu$ must be dense in $H$. It is obvious that the induced mapping of measures $i: M(H) \hookrightarrow M(G)$ is such that $\check{i} \nu=\mu$. That establishes (1), (2), and (3) when $\Gamma(\mu)$ is locally compact.

(4) This follows from Remark 10.1 $i H$ is the closed subgroup generated by the support of $i \nu=\mu$, and $\Gamma(\mu)=\Gamma(i \nu)=\widehat{H}$ is topologically isomorphic to $\widehat{G_{i H}} / H^{\perp}$, so $\mu$ is indeed a full measure in $M(i H)$.

Suppose now that $\tilde{\Gamma}(\mu)$ is locally compact. Let $H$ be the dual group of $\tilde{\Gamma}(\mu)$. We have $\Gamma \hookrightarrow \tilde{\Gamma}(\mu)$, and the image of $\Gamma$ (which is $\Gamma(\mu))$ is dense in $\tilde{\Gamma}(\mu)$, so the dual group mapping sends $H$ continuously and one-to-one into $G$. We now proceed as in the previous case. 
Here are some examples related to the preceding result. In the first example, the subgroup $H$ that Theorem 10.2 (2) says exists is the support group of the convolution of the product $\chi_{j=1}^{N} \mu_{j}$.

Remark 10.3. $\Gamma(\mu)$ is locally compact if $\mu=\sum_{j=1}^{N} \mu_{j}$, where the $\mu_{j}$ are mutually singular such that for each $j$ there exists a locally compact group topology $\tau_{j}$ on $G$ (stronger than the given one) such that $\mu_{j}$ is a full measure in $M\left(G_{\tau_{j}}\right)$.

Proof. This works because the sum is finite: embed $\Gamma(\mu)$ in $\prod_{j=1}^{N} \Gamma\left(\mu_{j}\right)$, using the mapping $\gamma \mapsto\left(\gamma_{1}, \ldots, \gamma_{N}\right)$, where $\gamma_{j}$ is the image of $\gamma$ in $\Gamma\left(\mu_{j}\right)$. That product space is locally compact since each factor is locally compact. Here are some of the details.

Suppose that $N=1$. Let $\mu$ be such that there exists a locally compact topology $\tau$ on $G$ such that $\mu \in M\left(G_{\tau}\right)$ and $\mu$ is full as a measure on $G_{\tau}$. Then $\Gamma\left(G_{\tau}, \mu\right)$ (in the obvious notation) is locally compact. But $\Gamma\left(G_{\tau}, \mu\right)=\Gamma(G, \mu)$. That proves the assertion for $N=1$, as well as showing that the product group $\prod_{j=1}^{N} \Gamma\left(\mu_{j}\right)$ is locally compact when $N>1$.

Since the $\mu_{j}$ are mutually singular, $L^{1}(\mu)$ is the direct sum

$$
\bigoplus \sum_{j=1}^{N} L^{1}\left(\mu_{j}\right)
$$

It follows easily that the image of $\Gamma(\mu)$ in $\prod_{j=1}^{N} \Gamma\left(\mu_{j}\right)$ is closed, and therefore locally compact. Indeed, the definition of the weak* topology on $\Gamma(\mu)$ and the mutual singularity of the $\mu_{j}$ imply that a net $\gamma_{\alpha}$ converges weak* in $\Gamma(\mu)$ iff $\gamma_{\alpha}$ converges weak* in $\Gamma\left(\mu_{j}\right)$ for each $j$. Therefore, $\Gamma(\mu) \rightarrow \prod_{j=1}^{N} \Gamma\left(\mu_{j}\right)$ is one-to-one and continuous. Since the diagonal $\{\gamma, \ldots, \gamma): \gamma \in \Gamma\}$ in $\prod_{j=1}^{N} \Gamma\left(\mu_{j}\right)$ is closed (the product being locally compact), $\Gamma(\mu)$ is (isomorphic to) a locally compact group.

It is not the case that $\Gamma(\mu)$ is locally compact iff $\mu=\sum_{1}^{\infty} \mu_{j}$, where the $\mu_{j}$ are mutually singular such that for each $j$ there exists a locally compact group topology $\tau_{j}$ on $G$ (stronger than the given one) such that $\mu_{j}$ is a full measure in $M\left(G_{\tau_{j}}\right)$. A counterexample to that is as follows.

Remark 10.4. Let $G=\prod_{j=1}^{\infty} G_{j}$, where each $G_{j}$ is a copy of the Bohr compactification of $\mathbb{R}$. Let $\mu_{j}$ be $2^{-j}$ times Lebesgue measure on $[0,1] \subset R$ (the copy in $G_{j}$ ) and $\mu=\sum \mu_{j}$. Then $\Gamma(\mu)=\sum \mathbb{R}$, the sum of an infinite number of copies of $\mathbb{R}$, which is not locally compact. The measure $\mu$ is Dirichlet.

Proof. The topology induced on $\Gamma$ by $\hat{\mu}$ is no weaker than that induced by the set $\left\{\hat{\mu}_{j}: 1 \leq j<\infty\right\}$. Since $\Gamma=\sum_{1}^{\infty} \mathbb{R}_{d}$, if a net $\gamma_{\alpha} \rightarrow \gamma$ in $\sum \mathbb{R}$, then $\gamma_{\alpha} \rightarrow \gamma$ in $\Gamma(\mu)$. Now, $\hat{\mu}\left(\gamma_{\alpha}\right)=\sum \hat{\mu}_{j}\left(\gamma_{\alpha}^{(j)}\right)$, where $\gamma_{\alpha}=\left(\gamma_{\alpha}^{(j)}\right)$, so $\hat{\mu}\left(\gamma_{\alpha}\right) \rightarrow \hat{\mu}(\gamma)$ if and only if $\hat{\mu}_{j}\left(\gamma_{\alpha}^{(j)}\right) \rightarrow \hat{\mu}_{j}\left(\gamma^{(j)}\right)$ for all $j$. Thus, convergence in $\Gamma(\mu)$ is the same as convergence in $\sum \mathbb{R}$.

To show that $\mu$ is Dirichlet, we let $\gamma^{(k)}$ be the character on $G=\Pi b \mathbb{R}$ that equals 1 (not the identity 0 ) in the $k^{\text {th }}$ factor and equals the identity in every other factor. Then $\gamma^{(k)} \rightarrow \infty$, while $\hat{\mu}\left(\gamma^{(k)}\right) \rightarrow\|\mu\|$. 
Saying $\Gamma(\mu)=\tilde{\Gamma}(\mu)$ is not quite the same as saying " $\mu$ is full in $G$ ". Let $G$ be the Bohr compactification of $R$, and let $\mu$ be Lebesgue measure on $[0,1]$ considered as a subset of $R \subset G$. Then $\mu$ is not full (because $\Gamma$ is discrete), but $\Gamma(\mu)=\tilde{\Gamma}(\mu)=R$ as locally compact abelian groups (though, of course, $\mu$ is full for the subgroup $i \mathbb{R} \hookrightarrow G)$.

Remark 10.5. $\tilde{\Gamma}(\mu)$ may be locally compact without being equal to $\Gamma(\mu)$. Indeed, let $G=T \times T$ and let $\mu$ be Lebesgue measure on [0,1], where we identify $R$ with the dense subgroup $H=\{(x, \sqrt{2} x):-\infty<x<\infty\}$ of $G$. Then $\Gamma(\mu)=Z \times Z$, while $\tilde{\Gamma}(\mu)=R$.

\section{Semigroup COMPaCtifications of NON-ABELIAN GROUPS}

We study some general compactifications of non-abelian locally compact groups.

Let $M$ be a von Neumann algebra and let $M_{*}$ be its unique predual. For each $x \in M, \phi \in M_{*}$, write $\left(L_{x} \phi\right)(y)=\phi(x y)$ and $\left(R_{x} \phi\right)(y)=\phi(y x), y \in M$. Then $L_{x} \phi, R_{x} \phi \in M_{*}$. We denote the unit ball in $M$ by $M_{1}$ and, of course, $|b|=\left(b^{*} b\right)^{1 / 2}$, $b \in M$. The various powers of $|b|$ belong to $M$ and are denoted either by $|b|^{q}$ or $\left(b^{*} b\right)^{q / 2}$.

Remarks 11.1. (i) For each $\phi \in M_{*}$,

$$
\left\{L_{x} \phi ; x \in M_{1}\right\} \text { and }\left\{R_{x} \phi ; x \in M_{1}\right\}
$$

are weakly compact subsets of $M_{*}$.

(ii) Any non-void weak ${ }^{*}$-closed subset $\Lambda$ of $M_{1}$ possesses an element $a$ such that $|a|$ is minimal in $|\Lambda|=\{|b|: b \in \Lambda\}$ in the ordering of $M^{+}$.

(iii) Let $\left\{x_{j}\right\}$ be a sequence in $M_{1}$ converging to a projection $h$ (a positive idempotent element). If $V$ is any neighborhood of $h$, there is a subsequence $\left\{x_{j_{\ell}}\right\}$ such that $V$ contains each product of the form $x_{j_{0}} x_{j_{1}}^{\epsilon_{1}} \ldots x_{j_{n}}^{\epsilon_{n}}$, for $n \geq 1$ and each choice $\epsilon_{1}=0, \pm 1 \ldots \epsilon_{n}=0, \pm 1$, where $x^{0}$ is defined to be $1, x^{1}=x$ and $x^{-1}=x^{*}$. If the orginal sequence is infinite, we may choose $\left\{x_{j_{\ell}}\right\}$ to be infinite. If the elements of the original sequence are distinct, then the products $x_{j_{0}} x_{j_{1}}^{\epsilon_{1}} \ldots x_{j_{n}}^{\epsilon_{n}}$ are all distinct.

Proof. (i) The map $x \rightarrow L_{x} \phi$ from $M$ to $M_{*}$ is weak*-weak continuous, and $M_{1}$ is compact in the weak ${ }^{*}$-topology. Hence $\left\{L_{x} \phi ; x \in M_{1}\right\}$ is weakly compact. Similarly $\left\{R_{x} \phi ; x \in M_{1}\right\}$ is also weakly compact.

(ii) Let $\Lambda^{\prime} \subset \Lambda$ be such that $\left|\Lambda^{\prime}\right|$ is totally ordered and give $\Lambda^{\prime}$ the order from $\left|\Lambda^{\prime}\right|$. In that ordering, $\Lambda^{\prime}$ possesses a weak* ${ }^{*}$-cluster point $\phi_{o} \in \Lambda$. If $\phi \in \Lambda^{\prime}$, then $\phi_{o}$ is a cluster point of the set $\left\{\psi \in \Lambda^{\prime}:|\psi| \leq|\phi|\right\}$. Hence $\phi_{o} \leq \phi$. An application of Zorn's lemma completes the argument.

(iii) This follows from an adaptation of [24, pp. 65-66].

Let $\sigma$ denote the weak*-topology on $M$. Let $1 \leq q<\infty$. By the $s_{q}$-topology on $M$ we shall mean the locally convex topology determined by the family of seminorms

$$
\left\{p_{\phi, q}: \phi \in M_{*} ; \phi \geq 0\right\}, \text { where } p_{\phi, q}(x)=\left(\phi\left(\left(x^{*} x\right)^{q / 2}\right)\right)^{1 / q} .
$$

Then, as is known, multiplication is separately continuous on $M$ when $M$ has the $\sigma$-topology and jointly continuous on the unit ball $M_{1}$ when $M_{1}$ has the $s_{2}$-topology; see Corollary 11.4 below, or [34, p. 18 and p. 21]. Hence $\left(M_{1}, \sigma\right)$ is a compact semitopological semigroup, and $\left(M_{1}, s_{1}\right)$ is a topological semigroup. Note that $\sigma \leq$ $s_{q}$ always. But $\sigma$ and $s_{q}$ do not agree on $M_{1}$ in general. 
The following extends known results for $\Delta M(G)$; see Lemma 2.6] and 24, p. 20, Lemme 7].

Proposition 11.2. Let $p \in M_{1}$ be a projection. Let $\Lambda=\Lambda(p)=\left\{x \in M_{1}: x^{*} x=\right.$ $p\}$. Let $\left\{\gamma_{\alpha}\right\}$ be a net in $\Lambda$ and $\gamma \in \Lambda$. Consider the following:

(1) $\gamma_{\alpha} \rightarrow \gamma$ weak*

(2) $\gamma_{\alpha} \rightarrow \gamma$ in the $s_{2}$-topology.

(3) $\gamma_{\alpha} \rightarrow \gamma$ in the $s_{1}$-topology.

(4) For some $1<q<\infty, \gamma_{\alpha} \rightarrow \gamma$ in the $s_{q}$-topology.

Then $(1) \Rightarrow(2)$, and (2)-(4) are equivalent.

Lemma 11.3. Let $x, y \in M$, and let $\mathcal{A}$ be a commutative $C^{*}$-subalgebra of $M$ which contains an identity $\mathbf{1}$. Let $\phi \in M^{*}, \phi \geq 0$, with $\phi(\mathbf{1})=1$ and $\|\phi\|=1$. Let $1<r<\infty$ and let $r^{\prime}$ be the conjugate index: $\frac{1}{r}+\frac{1}{r^{\prime}}=1$. Then the following hold for $x, y \in M$ :

$$
\begin{aligned}
\left|\phi\left(x^{*} x\right)\right| & \leq \phi\left(\left(x^{*} x\right)^{r}\right)^{1 / r} \phi\left(\mathbf{1}^{r^{\prime}}\right)^{1 / r^{\prime}}, \\
\phi\left(\left(x^{*} x\right)^{r / 2}\right) & \leq\|x\|^{r-1} \phi\left(x^{*} x\right)^{1 / 2} .
\end{aligned}
$$

Proof of Lemma 11.3. For (11.2): this is a version of the Hölder inequality, and the proof is by using the $C^{*}$-algebra generated by $x^{*} x$ and $\mathbf{1}$. Then $\phi$ restricts to a probability measure on that $C^{*}$-algebra. The proof then proceeds as usual for measurable spaces [6, p. 120].

For (11.3): use the $C^{*}$-algebra generated by $x^{*} x$ and $\mathbf{1}$. Then $\phi$ restricts to a probability measure on that $C^{*}$-algebra. The proof then proceeds as usual for measurable spaces.

Proof of Proposition 11.2. As in the proof of Lemma 2.6, we will show that the semi-norms $p_{\phi, q}, 1 \leq p<\infty$, are equivalent on $\Lambda$. For this proof, it will be enough to consider only those $\phi \geq 0$ with $\phi(p)=1$.

$(1) \Rightarrow(2)$. By the assumptions on $\phi, \phi\left(\left(\gamma-\gamma_{\alpha}\right)^{*}\left(\gamma-\gamma_{\alpha}\right)\right)=2-2 \operatorname{Re} \phi\left(\gamma^{*} \gamma_{\alpha}\right)$. Then weak* convergence in $M$ implies

$$
\phi\left(\gamma^{*} \gamma_{\alpha}\right) \rightarrow \phi\left(\gamma^{*} \gamma\right)=1,
$$

and (2) follows.

$(2) \Rightarrow(3)$ is immediate from the Cauchy-Schwarz-Buniakowski inequality:

$$
\begin{aligned}
\phi\left(\left(\left(\gamma-\gamma_{\alpha}\right)^{*}\left(\gamma-\gamma_{\alpha}\right)\right)^{1 / 2}\right) & =\left(\phi\left(\left(\gamma-\gamma_{\alpha}\right)^{*}\left(\gamma-\gamma_{\alpha}\right)\right)^{1 / 2} p\right) \\
& \leq\left(\phi\left(\left(\gamma-\gamma_{\alpha}\right)^{*}\left(\gamma-\gamma_{\alpha}\right)\right)\right)^{1 / 2}\left(\phi\left(p^{2}\right)\right)^{1 / 2} \\
& =\left(\phi\left(\left(\gamma-\gamma_{\alpha}\right)^{*}\left(\gamma-\gamma_{\alpha}\right)\right)\right)^{1 / 2} .
\end{aligned}
$$

(3) $\Rightarrow(4)$. Let $1<p<\infty$. Then

$$
\begin{aligned}
& \phi\left(\left(\left(\gamma-\gamma_{\alpha}\right)^{*}\left(\gamma-\gamma_{\alpha}\right)\right)^{p / 2}\right) \\
& =\phi\left(\left(\left(\gamma-\gamma_{\alpha}\right)^{*}\left(\gamma-\gamma_{\alpha}\right)\right)^{(p-1) / 2}\left(\left(\gamma-\gamma_{\alpha}\right)^{*}\left(\gamma-\gamma_{\alpha}\right)\right)^{1 / 2}\right) \\
& \quad \leq 2^{p-1} \phi\left(\left(\left(\gamma-\gamma_{\alpha}\right)^{*}\left(\gamma-\gamma_{\alpha}\right)\right)^{1 / 2}\right) .
\end{aligned}
$$

$(4) \Rightarrow(2)$. This is immediate from (11.2). 
Corollary 11.4. Let $\Lambda=\Lambda(p)$ and let $p$ be as in Proposition 11.2. Then the multiplication in $\Lambda$ is continuous in both variables simultaneously under any of the topologies (2)-(4) of Proposition 11.2 .

In the context of von Neumann algebras, we have the following analogue of Lemma 2.5.

Proposition 11.5. Let $U$ denote the group of units in $M_{1}$ (the largest subgroup containing 1$)$. Then the map $M_{1} \times M_{1} \rightarrow M_{1},(x, y) \rightarrow x y$ is jointly continuous at each $(x, u)$ and $(u, x)$, for $x \in M_{1}, u \in U$, when $M_{1}$ has the $\sigma$-topology, and in the $s_{q}$-topologies.

Proof. This follows from the Ellis-Lawson Joint continuity theorem; see [33, p. 95] for the $\sigma$-topology, and Proposition 11.2 for the $s_{q}$-topologies.

Remark 11.6. The $s_{q}$-topologies $(1 \leq q<\infty)$ and the $\sigma$-topology agree on the set of projections in $M_{1}$. (This is well known: assume the $\left\{p_{\alpha}\right\}$ is a net of projections, and $p_{\sigma}{ }^{\sigma} \rightarrow p, p$ a projection. Then for any $\phi \in M_{*}, \phi \geq 0$,

$$
\begin{aligned}
\phi\left(\left(p_{\alpha}-p\right)^{*}\left(p_{\alpha}-p\right)\right) & =\phi\left(\left(p_{\alpha}-p\right)\left(p_{\alpha}-p\right)\right) \\
& =\phi\left(p_{\alpha}\right)-\phi\left(p p_{\alpha}\right)-\phi\left(p_{\alpha} p\right)+\phi(p) \\
& \rightarrow 0
\end{aligned}
$$

by separate continuity in the $\sigma$-topology. Hence the $\sigma$-topology and the $s_{2}$-topologies agree. Now apply the Cauch-Schwarz-Buniakowski inequality and (11.3) to obtain the equivalance with the $s_{q}$-topologies.

Let $G$ be a locally compact group and let $M$ be a von Neumann algebra. Let $\rho$ : $G \rightarrow M$ be a continuous homomorphism of $G$ into the group of unitary elements in $M$ with the relative weak* (i.e. $\sigma$ )-topology. For each $\phi \in M_{*}$, let $\hat{\phi}(g)=\phi(\rho(g))$, for $g \in G$. Then clearly $\hat{\phi}$ is a bounded continuous function on $G$. Furthermore if $a \in G$, then $f_{a}=\left(L_{\rho(a)} \phi\right)^{\wedge}$. Now the map $\phi \rightarrow \hat{\phi}$ from $M_{*}$ into $C B(G)$ is weak-weak continuous. Using that and Remarks 11.1(i), we conclude that each $\hat{\phi} \in W A P(G)$, the space of continuous weakly almost periodic functions on $G$. Let $\overline{\rho(G)}$ denote the closure of $\rho(G)$ in $M$ in the $\sigma$-topology. Then $\overline{\rho(G)}$ with the $\sigma$-topology is a compact semitopological semigroup, and $\rho: G \rightarrow \overline{\rho(G)}$ is a continuous embedding of $G$ into a dense subset of $\overline{\rho(G)}$. Hence if $\bar{G}^{w}$ denotes the weakly almost periodic compactification of $G$, and $i: G \rightarrow \bar{G}^{w}$ is the embedding of $G$ in $\bar{G}^{w}$, then there exists a continuous homomorphism $\gamma$ from $\bar{G}^{w}$ onto $\overline{\rho(G)}$ such that the following diagram commutes (see [2]):

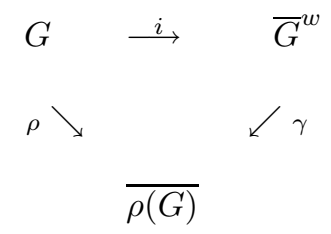

Let $N$ be the $\sigma$-closure of the linear span of $\overline{\rho(G)}^{\sigma}$, the von Neumann subalgebra in $M$ generated by $\rho(G)$. Then $\overline{\rho(G)} \subseteq N$. Since $N_{*}=\left\{\phi_{\mid N}: \phi \in M_{*}\right\}$ (see [31, p. 317]) the $\sigma\left(N, N_{*}\right)$ and $\sigma\left(M, M_{*}\right)$ topologies on $N$ are the same. Hence we shall from now on assume that $N=M$. 
Proposition 11.7. For each $x \in \overline{\rho(G)}$, the restriction of the multiplication of $\overline{\rho(G)}$ to $x \overline{\rho(G)} \times \overline{\rho(G)}$ is jointly continuous at $(x, s)$, for each $s \in \overline{\rho(G)}$ in the $\sigma$-topology and in the $s_{q}$-topologies.

Proof. $\sigma$-topology: this follows from the fact that the group of units in $\overline{\rho(G)}$ (which contains $\rho(G))$ is dense in $\overline{\rho(G)}$ and [33, p. 70].

$s_{q}$-topologies: immediate from Proposition 11.2 .

Corollary 11.8. Let 1 be the identity in $M$. Then the multiplication in $\overline{\rho(G)}$ is jointly continuous at $(1,1)$ in the $\sigma$-topology and the $s_{q}$-topologies (note that $\rho(e)=1)$.

Corollary 11.9. The identity map $i$ is continuous from $(\overline{\rho(G)}, \sigma)$ to $\left(\overline{\rho(G)}, s_{2}\right)$ at 1 in the $\sigma$ and $s_{q}$-topologies.

Proof. Let $x_{\alpha} \in \overline{\rho(G)}$ such that $x_{\alpha} \stackrel{\sigma}{\longrightarrow} 1$. Let $\phi \in M_{*}, \phi \geq 0$. Then

$$
\phi\left(\left(x_{\alpha}-1\right)^{*}\left(x_{\alpha}-1\right)\right)=\phi\left(x_{\alpha}^{*} x_{\alpha}\right)-\phi\left(x_{\alpha}^{*}\right)-\phi\left(x_{\alpha}\right)+\phi(1) \rightarrow 0
$$

by Corollary 11.8. It follows that $\phi\left(\left|x_{\alpha}-1\right|\right) \rightarrow 0$. Hence, we have the limit in $\sigma$ (weak*). Now apply Lemma 2.6.

For each $\phi \in M_{*}$, let $\mathcal{L} O^{(*)}(\phi)=\left\{L_{\rho(g)} \phi ; g \in G\right\}$ and let $\mathcal{R} O^{(*)}(\phi)=\left\{R_{\rho(g)} \phi ;\right.$ $g \in G\}$. By Remarks 11.1(i), both $\mathcal{L} O^{(*)}(\phi)$ and $\mathcal{R} O^{(*)}(\phi)$ are relatively weakly compact subsets of $M_{*}$. For $\phi \in M_{*}$, define a seminorm of $M$ by

$$
n_{\phi}(x)=\sup \{|\phi(\rho(g) x)| ; g \in G\} \text {. }
$$

Let $\tau$ be the locally convex topology on $M$ determined by all the seminorms $\left\{n_{\phi}: \phi \in M_{*}\right\}$. Then, clearly $\tau$ is stronger than $\sigma$. Also by the Mackey-Arens Theorem, $\tau$ is a topology of the dual pair $\left(M, M_{*}\right)$, i.e. the continuous linear functionals on $M$ with respect to $\tau$ are also the set $M_{*}$.

Proposition 11.10. Let $G$ be as above. Then the following hold:

(1) The action of $G$ on $\overline{\rho(G)}$, defined by $(g, x) \rightarrow \rho(g) x, g \in G, x \in \overline{\rho(G)}$, is jointly continuous when $\overline{\rho(G)}$ has the $\sigma$-topology.

(2) The action above is equicontinuous when $\overline{\rho(G)}$ has the $\tau$-topology.

(3) $(\rho(G), \sigma)$ is a topological group.

Proof. The first and last items follow from Proposition 11.5.

The second follows from the simple observation that $p_{\phi}(g \cdot x)=p_{\phi}(x)$ for each $\phi \in M_{*}, g \in G$.

Remark 11.11. It is easy to see that $\overline{\rho(G)}$ has jointly continuous multiplication in the $\sigma$-topology if and only if $\overline{\rho(G)}$ is a compact group.

Let $\overline{\rho(G)}^{+}$denote the set of positive elements in $\overline{\rho(G)}$.

Proposition 11.12. $\left(\overline{\rho(G)}^{+}, \sigma\right)$ is compact, contains the identity element of $M$ and for each $x \in \overline{\rho(G)}, \quad|x|^{2}=x^{*} x \in \overline{\rho(G)}^{+}$. In particular if $p \in \overline{\rho(G)}$ is a projection, then $p \in \overline{\rho(G)}^{+}$. 
Proof. Since $\overline{\rho(G)}^{+}=: \overline{\rho(G)} \cap M^{+}$, and $M^{+}$is $\sigma$-closed [34, p. 14], $\overline{\rho(G)}^{+}$must be compact in the $\sigma$ topology. Also, if $x \in \overline{\rho(G)}$, let $\left\{\rho\left(g_{\alpha}\right)\right\}$ be a net converging to $x$ in the $\sigma$-topology; then $\rho\left(g_{\alpha}\right)^{*}=\rho\left(g_{\alpha}^{-1}\right) \rightarrow x^{*}$ in the $\sigma$-topology. Hence $x^{*} \in \overline{\rho(G)}$. So $x^{*} x \in \overline{\rho(G)}$ and $x^{*} x \geq 0$, i.e. $x^{*} x \in \overline{\rho(G)}^{+}$.

Proposition 11.13. If $\overline{\rho(G)}$ consists of partial isometries, then the set of projections in $\overline{\rho(G)}, \overline{\rho(G)}$ forms a commutative semitopological semigroup.

Proof. We may assume that $M$ acts on a Hilbert space $H$. Now if $p, q \in \overline{\rho(G)}$, then $p q, q p \in \overline{\rho(G)}$. Hence they are partial isometries. Now if $\xi \in H$, then $\|p q(\xi)\|=$ $\|\xi\| \Longleftrightarrow q \xi=\xi=p \xi$ and so $p q \xi=\xi$. (Indeed, if $\|p q \xi\|=\|\xi\|$, then $\|q(\xi)\| \geq$ $\|p q(\xi)\|=\|\xi\|$. So $\|q(\xi)\|=\|\xi\|$, i.e. $q(\xi)=\xi$. Consequently, $\|p q(\xi)\|=\|p(\xi)\|=$ $\|\xi\|$, i.e. $p(\xi)=\xi$.) Similarly $\|p q(\xi)\|=\|\xi\| \Longleftrightarrow p(\xi)=\xi=q(\xi)$ and so $q p(\xi)=\xi$. Hence $p q=q p$. Also $(p q)^{*}=p q$ and $(p q)^{2}=p q$, i.e. $\left.p q \in \overline{\rho(G)}\right)_{e}$. Hence $\overline{\rho(G)} e_{e}$ is a commutative semigroup.

Corollary 11.14. If $\overline{\rho(G)}^{+}$consists of projections, then $\overline{\rho(G)}^{+}$is a compact commutative topological semigroup.

Proof. In this case, for each $x \in \overline{\rho(G)}, x^{*} x \in \overline{\rho(G)}^{+}$. Hence $x$ is a partial isometry.

To see that multiplication on $\overline{\rho(G)}^{+}$is jointly continuous, we note that the WOT (weak operator topology) agrees with the $\sigma$-topology on $\overline{\rho(G)}^{+}$since $\sigma$ is stronger than the WOT; $\overline{\rho(G)}^{+}$is $\sigma$-compact and WOT is Hausdorff. Also the WOT agrees with the $S O T$ (strong operator topology) on $\overline{\rho(G)}^{+}$since $\overline{\rho(G)}^{+}$consists of projections. Hence $\sigma$-topology and the SOT agree on $\overline{\rho(G)}^{+}$, and multiplication is jointly continuous with respect to the $S O T$ as readily checked.

\section{Problems}

(1) For $f \in A_{p}(G)$, is it the case that $\left\{\delta_{x} * f: x \in G\right\}$ is relatively weakly compact iff either $f=0$ or $G$ is compact?

(2) For $f \in A_{p}(G)$ and abelian $G$, is it the case that $\{\gamma f: \gamma \in \widehat{G}\}$ is relatively weakly compact iff $f=0$ or the dual group $\widehat{G}$ is compact? The proof of Proposition 6.2 says the answer is "Yes" if there is a Littlewood-Paley theory; what about the general case?

(3) For which $L^{p}$-multipliers $M$ on $\mathbb{T}$ is $\{\gamma M: \gamma \in \mathbb{Z}\}$ relatively weakly compact? What if $\widehat{M}$ vanishes at infinity but is not in the closure of the trigonometric polynomials? See Proposition 6.5.

(4) For which measures $\mu \in M(\mathbb{T})$ is it the case that $\left\{\delta_{x} * \mu: x \in \mathbb{T}\right\}$ is relatively weakly compact in $M_{p}(\mathbb{T})$ ?

(5) Let $U_{\alpha}$ be a weakly convergent net of unitary elements of a von Neuman algebra. Is the limit element unitary?

(6) Let $G$ be a compact group with Fourier algebra $A(G)$ and von Neumann algebra $V N(G)=A(G)^{*}$. If $S \in V N(G)$ is such that $O^{(*)}(S)=\left\{\delta_{x} * S\right.$ : $x \in G\}$ is relatively weakly compact, is $S$ in the norm closure of $L^{1}(G)$ in $V N(G)$ ? (The converse is evident: $O^{(*)}(S)$ is norm compact.)

(7) Let $\{\rho, H\}$ be a continuous unitary representation of $G$. What is the structure of $\overline{\rho(G)} \subset \mathcal{B}(H)$ in terms of $\rho$ ? 
(8) Is there a theory for relatively weak* compact sets?

(9) If $G$ is a locally compact group and $P \in V N(G)$ is a projection and a net of left translates $L_{x_{\alpha}} P \rightarrow T$ weakly, what can we say about $T$ ?

(10) Does $\mu$ tame $\Rightarrow \Gamma(\mu)$ is locally compact?

(11) What is the structure of $\tilde{\Gamma}(\mu) / \Gamma(\mu)$ ?

(12) What are the idempotents in $\bar{\Gamma}(\mu)$ ?

(13) What are the minimal ideals in $\bar{\Gamma}(\mu)$ ?

\section{REFERENCES}

[1] L. N . Argabright and J. Gil de Lamadrid, Almost periodic measures, Memoirs Amer. Math. Soc. 85, no. 485 (1990). MR0979431 (90k:43001)

[2] J. Berglund, H. Junghenn and Paul Milnes, Analysis on Semigroups, Wiley-Interscience-Canadian Math. Society, New York (1989). MR0999922 (91b:43001)

[3] G. Brown, Riesz products and generalized characters, Proc. London Math. Soc. (3) 27 (1973), 484-504. MR0372530 (51:8737)

[4] G. Brown, C. C. Graham, and W. Moran, Translation and symmetry in $M(G)$, Symposia Mathematica XXII (1977), 371-392. MR0487272 (58:6920)

[5] R. B. Burckel, Weakly almost periodic functions on semigroups, Gordon and Breach, New York, N. Y. (1970). MR0263963(41:8562)

[6] N. Dunford and and J. T. Schwartz, Linear operators, Part I: General Theory, Interscience Publishers, New York, N. Y. (1957). MR1009162 (90g:47001a)

[7] C. Dunkl and D. E. Ramirez, Sections induced from weakly sequentially complete spaces, Studia Mathematica 59 (1973), 95-97. MR0333591(48:11916)

[8] M. Déchamps-Gondim, Ensembles de Sidon topologiques, Ann. Inst. de Fourier (Grenoble) 22 (fasc. 3) (1972), 51-79. MR0340981 (49:5731)

[9] W. F. Eberlein, Abstract ergodic theorems and weak almost periodic functions, Transactions of the Amer. Math. Soc. 67 (1949), 217-240. MR0036455 (12:112a)

[10] A note on the Fourier-Stieltjes transform, Proceedings of the Amer. Math. Soc. 6 (1953), 310-312. MR0068030(16:817b)

[11] R. E. Edwards and G. I. Gaudry, Littlewood-Paley and Multiplier Theory, Springer-Verlag, New York (1977). MR0618663(58:29760)

[12] P. Eymard, L'algébre de Fourier d'un groupe localement compact, Bull. Soc. Math. France 92 (1964), 181-236. MR0228628 (37:4208)

[13] J. Galindo and S. Hernández, The concept of boundedness and the Bohr compactification of a MAP abelian group, Fund. Math. 15, no. 3 (1999), 195-218. MR1680642 (2001c:22001)

[14] C. C. Graham, Arens regularity for quotients of the Herz algebra $A_{p}$, Bull. London Math. Soc. 34 (2002), 457-468. MR1897425 (2003h:43003)

[15] C. C. Graham and O. Carruth McGehee, Essays in Commutative Harmonic Analysis Springer-Verlag, New York (1979). MR0550606 (81d:43001)

[16] K. E. Hare and L. T. Ramsey, $I_{0}$ sets in non-abelian groups, Math. Proc. Cambridge Phil. Soc. 135 (2003), 81-98. MR.1990833 (2004e:43011)

[17] S. Hartman and C. Ryll-Nardzewski, Almost periodic extensions of functions, I, Colloq. Math. 12 (1964), 23-29. MR0167785(29:5057)

[18] E. Hewitt and K. A. Ross, Abstract Harmonic Analysis, vol. I, Springer-Verlag, Berlin (1963). MR0156915 (28:158)

[19] E. Hewitt and K. A. Ross Abstract Harmonic Analysis, vol. II, Springer-Verlag. Berlin (1970). MR0262773 (41:7378)

[20] C. Herz, Harmonic synthesis for subgroups, Ann. Inst. Fourier (Grenoble) 23, fasc. 3 (1973), 91-123. MR0355482(50:7956)

[21] C. Herz, Une generalization de la notion de transformée de Fourier-Stieltjes, Ann. Inst. Fourier (Grenoble) 24, fasc. 3 (1974), 145-157. MR0425511 (54:13466)

[22] E. Hewitt and K. Stromberg, Real and Abstract Analysis, Springer-Verlag, Berlin (1965). MR0188387 (32:5826)

[23] B. Host, Le théorème des idempotents dans $B(G)$, Bull. Soc. Math. France 114 (1986), 215-223. MR0860817 (88b:43003) 
[24] B. Host, J.-F. Mela, and F. Parreau, Analyse Harmonique des Measures, Société Mathématique de France, Paris (1986). MR0839692 (88a:43005)

[25] B. Host, J.-F. Mela, and F. Parreau, Non-singular transformations and spectral analysis of measures, Bull. Math. Soc. France (1991), 33-90. MR.1101939 (93d:43002)

[26] K. Kunen and W. Rudin, Lacunarity and the Bohr topology, Math. Proc. Camb. Phil. Soc. 126 (1999), 117-137. MR.1681658 (2000e:43003)

[27] A. T.-M. Lau and J. C. S. Wong, Weakly almost periodic elements in $L^{\infty}(G)$ of a locally compact group, Proceedings of the Amer. Math. Soc. 107 (1989), 1031-1036. MR0991701 (90m:43006)

[28] J. M. López and K. A. Ross, Sidon Sets, Marcel Dekker, New York (1975). MR0440298 $(55: 13173)$

[29] J.-F. Méla, Suites lacunaires de Sidon, ensembles propre et points exceptionnels, Ann. Inst. de Fourier (Grenoble) 14 (fasc. 2) (1964), 533-538. MR.0178308 (31:2566)

[30] _ Sur certains ensembles exceptionnels en analyse de Fourier, Ann. Inst. de Fourier (Grenoble) 18 (1968), 32-71. MR0412739 (54:860)

[31] J. R. Ringrose, Lectures on the trace in a finite von Neumann algebra, Lectures on operator algebras, Lecture Notes in Mathematics \#247, Springer-Verlag (1972), 309-354. MR0361816 (50:14261)

[32] W. Rudin, Fourier Analysis on Groups, Wiley Interscience, New York (1962). MR0152834 $(27: 2808)$

[33] W. Ruppert, Compact semitopological semigroups: an intrinsic theory, Lecture Notes in Mathematics \#1079, Springer-Verlag, Berlin, Heidelberg, New York, Tokyo (1984). MR0762985 (86e:22001)

[34] S. Sakai, $C^{*}$-algebras and $W^{*}$-algebras, Springer-Verlag, New York, Heidelberg, Berlin (1971). MR0442701 (56:1082)

Department of Mathematics, University of British Columbia, RR \#1 - D-156, Bowen Island, British Columbia, CAnada V0N 1G0

E-mail address: ccgraham@alum.mit.edu

Department of Mathematical and Statistical Sciences, University of Alberta, Edmonton, Alberta, Canada T6G 2G1

E-mail address: tlau@math.ualberta.ca 